\title{
BLAZARS IN THE FERMI ERA: THE OVRO 40 m TELESCOPE MONITORING PROGRAM
}

\author{
Joseph L. Richards ${ }^{1}$, Walter Max-Moerbeck ${ }^{1}$, Vasiliki Pavlidou ${ }^{1,11}$, Oliver G. King ${ }^{1}$, Timothy J. Pearson ${ }^{1}$, \\ Anthony C. S. Readhead ${ }^{1}$, Rodrigo Reeves ${ }^{1}$, Martin C. Shepherd ${ }^{1}$, Matthew A. Stevenson ${ }^{1}$, Lawrence C. Weintraub ${ }^{1}$, \\ Lars Fuhrmann ${ }^{2}$, Emmanouil Angelakis ${ }^{2}$, J. Anton Zensus ${ }^{2}$, Stephen E. Healey ${ }^{3}$, Roger W. Romani ${ }^{3}$, \\ Michael S. Shaw ${ }^{3}$, Keith Grainge ${ }^{4,5}$, Mark Birkinshaw ${ }^{6}$, Katy Lancaster $^{6}$, Diana M. Worrall ${ }^{6}$, Gregory B. TaYlor ${ }^{7}$, \\ GARRET COTTER $^{8}$, AND RiCARDO Bustos ${ }^{9,10}$ \\ ${ }^{1}$ Cahill Laboratory of Astronomy and Astrophysics, California Institute of Technology, 1200 E California Blvd, Pasadena, CA 91125, USA; joey@ caltech.edu \\ ${ }^{2}$ Max-Planck-Institut-für-Radioastronomie, Auf dem Hügel 69, 53121 Bonn, Germany \\ ${ }^{3}$ Department of Physics, Stanford University, Stanford, CA 94305, USA \\ ${ }^{4}$ Astrophysics Group, Cavendish Laboratory, University of Cambridge, J J Thomson Ave, Cambridge CB3 OHE, UK \\ ${ }^{5}$ Kavli Institute for Cosmology Cambridge, Madingley Road, Cambridge CB3 OHA, UK \\ ${ }^{6}$ H. H. Wills Physics Laboratory, University of Bristol, Tyndall Ave, Bristol BS8 1TL, UK \\ ${ }^{7}$ Department of Physics and Astronomy, University of New Mexico, Albuquerque, NM 87131, USA \\ ${ }^{8}$ Department of Astrophysics, University of Oxford, Keble Road, Oxford, OX1 3RH, UK \\ ${ }^{9}$ Departamento de Astronomía, Universidad de Concepción, Casilla 160-C, Concepción, Chile \\ ${ }^{10}$ Departamento de Astronomía, Universidad de Chile, Casilla 36-D, Santiago, Chile \\ Received 2010 November 12; accepted 2011 March 22; published 2011 May 23
}

\begin{abstract}
The Large Area Telescope (LAT) aboard the Fermi Gamma-ray Space Telescope provides an unprecedented opportunity to study gamma-ray blazars. To capitalize on this opportunity, beginning in late 2007, about a year before the start of LAT science operations, we began a large-scale, fast-cadence $15 \mathrm{GHz}$ radio monitoring program with the $40 \mathrm{~m}$ telescope at the Owens Valley Radio Observatory. This program began with the 1158 northern $\left(\delta>-20^{\circ}\right)$ sources from the Candidate Gamma-ray Blazar Survey and now encompasses over 1500 sources, each observed twice per week with about 4 mJy (minimum) and 3\% (typical) uncertainty. Here, we describe this monitoring program and our methods, and present radio light curves from the first two years (2008 and 2009). As a first application, we combine these data with a novel measure of light curve variability amplitude, the intrinsic modulation index, through a likelihood analysis to examine the variability properties of subpopulations of our sample. We demonstrate that, with high significance $(6 \sigma)$, gamma-ray-loud blazars detected by the LAT during its first 11 months of operation vary with almost a factor of two greater amplitude than do the gamma-ray-quiet blazars in our sample. We also find a significant $(3 \sigma)$ difference between variability amplitude in BL Lacertae objects and flat-spectrum radio quasars (FSRQs), with the former exhibiting larger variability amplitudes. Finally, low-redshift $(z<1)$ FSRQs are found to vary more strongly than high-redshift FSRQs, with $3 \sigma$ significance. These findings represent an important step toward understanding why some blazars emit gamma-rays while others, with apparently similar properties, remain silent.
\end{abstract}

Key words: BL Lacertae objects: general - galaxies: active - methods: statistical - quasars: general - radio continuum: galaxies

Online-only material: figure set, machine-readable tables

\section{INTRODUCTION}

The rotating supermassive black holes that power active galactic nuclei (AGNs) somehow accomplish the remarkable feat of channeling energy derived from their rotation and accretion disks into two relativistic jets oppositely directed along the spin axis. In spite of intensive observational efforts over the last four decades, the detailed mechanism of this process has remained elusive, and, although several processes have been suggested, we are still largely ignorant of the composition of the jets and the forces that collimate them. The first detailed collimation mechanism to be proposed was that of a "de Laval" nozzle (Blandford \& Rees 1974), which is now known to be a likely cause of re-collimation on kpc scales, but not of the initial collimation, which, as revealed by Very Long Baseline Interferometry (VLBI), clearly occurs on sub-parsec scales. Other early theories, which involve magnetohydrodynamic winds (Blandford \& Znajek 1977) and/or magnetic fields

\footnotetext{
${ }^{11}$ Einstein fellow.
}

threading the inner accretion disk (Blandford \& Payne 1982), remain the most promising approaches to a full understanding of the phenomenon.

An observational difficulty is that, except in a few cases (e.g., M87), radio observations, which provide the most detailed images of active galaxies, only probe the relativistic jets down to the point at which the jets become optically thick at a point some light-weeks or light-months from the site of the original collimation. Higher-frequency observations are needed to probe deeper into the jets, although interstellar scintillation observations do in some cases reveal the presence of radio emission features in some AGNs that are $\sim 5-50 \mu$ as in extent (Kedziora-Chudczer et al. 1997; Dennett-Thorpe \& de Bruyn 2000; Jauncey et al. 2000; Rickett et al. 2002, 2006; Lovell et al. 2008), which can be very persistent (Macquart $\&$ de Bruyn 2007). These mysterious, very high brightness temperature features are by no means understood and are certainly of great interest. At optical wavelengths, rapid swings in the polarization position angle have been used to tie together flux density variations at $\mathrm{TeV}$ energies and variations at millimeter wavelengths (Marscher et al. 
2008). At very high energies of hundreds of GeV to TeV, very rapid variations down to timescales of minutes have been observed by the HESS, MAGIC, and VERITAS instruments (e.g., Aharonian et al. 2007; Aharonian et al. 2009; Acciari et al. 2009; Acciari et al. 2010). Full three-dimensional (non-axisymmetric) magnetohydrodynamic relativistic simulations are now being carried out that enable detailed interpretation of the observations over the whole electromagnetic spectrum (e.g., McKinney \& Blandford 2009; Penna et al. 2010).

Relativistic beaming introduces complications in observational studies of relativistic jets. The continuum emission is strongly beamed along the jet axis, introducing strong observational selection effects. Those objects having jets that are aligned at a small angle to the line of sight are collectively known as "blazars." Small variations in the angle between the jet axis and the line of sight result in a large range of observed properties, such as apparent luminosity, variability, and energy spectrum. Strong boosting of the continuum synchrotron emission from the jet also frequently swamps optical line emission, making it difficult or even impossible to obtain a redshift for the source. As a result, blazars are subdivided into two classes: flat-spectrum radio quasars (FSRQs) and BL Lacertae objects (BL Lacs). The former class contains blazars dominated by strong broad emission lines while the latter class contains those blazars with spectra dominated by their continuum emission, and hence weak, if any, emission lines and very weak absorption lines, or no lines at all. The large variations in the energy spectrum make it difficult to study many blazars over the whole electromagnetic spectrum. As a result the study of large, carefully selected samples is necessary to determine the physical processes and conditions of the parent population. As relativistically boosted emission can be detected even from high-redshift sources, any intrinsic scatter in jet properties and scatter due to relativistic beaming is additionally convolved with any cosmological evolution of the black holes giving rise to the jets and their environment. It is therefore not surprising that the study of the population properties of relativistic jets has, to this day, been sparse at best.

The launch of the Fermi Gamma-ray Space Telescope in June of 2008 provides an unprecedented opportunity for the systematic study of blazar jets (Atwood et al. 2009). Its Large Area Telescope (LAT) observes the sky at energies between $100 \mathrm{MeV}$ and a few hundred GeV. In this energy range relativistic particles can be probed through their inverse Compton emission in the case of electron/positron jets (e.g., Dermer et al. 1992; Sikora et al. 1994; Blandford \& Levinson 1995), or a combination of pionic emission from primaries and inverse Compton emission from cascade-produced leptonic secondaries in the case of hadronic jets (e.g., Mannheim 1993).

Blazars comprise the most numerous class of extragalactic $\mathrm{GeV}$ sources associated with lower-energy counterparts: the first-year Fermi point-source catalog (1FGL) contains 1451 sources, of which 596 have been associated with blazars in the first AGN catalog (1LAC; Abdo et al. 2010a, 2010b). As the LAT completes one survey of the whole sky every $3 \mathrm{hr}$, it can provide continuous monitoring of all gamma-ray bright blazars, although with variable cadence that depends on the integration time necessary to detect each object (which can range from a single satellite pass to many months, depending on the average flux density of the object and its activity state).

The exact location of the gamma-ray emission region and its proximity to the central black hole remain subjects of debate. Two possible models of the $\mathrm{GeV}$ emission region are that this emission comes from a "gamma-sphere" close to the base of the jet (Blandford \& Levinson 1995) or that it comes from the same shocked regions that are responsible for the radio emission seen in VLBI observations much further out in the jet (Jorstad et al. 2001). If the former model is correct then the gamma-ray observations might well provide evidence of the initial collimation mechanism.

The testing of models of the location, structure, and radiative properties of the gamma-ray emission region in blazars requires, in addition to the Fermi observations, supporting broadband observations of likely gamma-ray sources in various activity states. Such multiwavelength efforts can occur in two modes:

1. regular monitoring of a preselected, statistically complete sample of likely gamma-ray-bright objects, independent of their gamma-ray activity state and

2. intensive observations of archetypal objects or objects exhibiting unusual behavior.

The blazar monitoring program we discuss here is focused on the first mode. In anticipation of the unique opportunities offered by the Fermi-LAT sky monitoring at gamma-ray energies, three years ago we began the bi-weekly monitoring of a large sample including 1158 likely gamma-ray-loud blazars, preselected according to uniform criteria, with the Owens Valley Radio Observatory (OVRO) $40 \mathrm{~m}$ telescope at $15 \mathrm{GHz}$. We also apply our observations in studies of the second mode through Fermi-LAT multiwavelength campaigns for flaring sources (e.g., Abdo et al. 2009; Fermi-LAT Collaboration et al. 2010) and through collaboration with the F-GAMMA project, a complementary effort representing the second mode, focused on radio and submillimeter spectral monitoring of about 60 prominent sources (Angelakis et al. 2010; Fuhrmann et al. 2007).

The sample that we are studying with the OVRO $40 \mathrm{~m}$ telescope is statistically well defined and large enough to allow for statistical analyses and comparisons of sub-samples. In addition, as the $40 \mathrm{~m}$ telescope is dedicated full time to this project, the cadence is high enough to allow sampling of the radio light curves on timescales comparable with those typically achieved by Fermi-LAT for bright gamma-ray blazars and in this sense the $40 \mathrm{~m}$ and Fermi-LAT are ideally matched. The combination of sample size and cadence is unprecedented, making this by far the largest monitoring survey of radio sources that has been undertaken as of the date of writing.

Data from this program, in combination with Fermi observations, will allow us systematically to derive the radio and radio/ gamma ray observational properties of the blazar population, including

1. the radio variability properties of the blazar population, their dependence on redshift, spectral classification, luminosity, and gamma-ray activity;

2. any differences between the radio properties of gammaray-loud blazars and blazars with similar radio luminosity which have not been detected by Fermi;

3. the properties (e.g., significance of correlation and the length and sign of any time delays) of cross-correlations between radio and gamma-ray flares of gamma-ray-loud blazars; and

4. the combination of radio properties, if one exists, that can predict the apparent gamma-ray luminosity of a blazar (which, in turn, could be used to derive blazar gamma-ray luminosity functions from radio luminosity functions). 


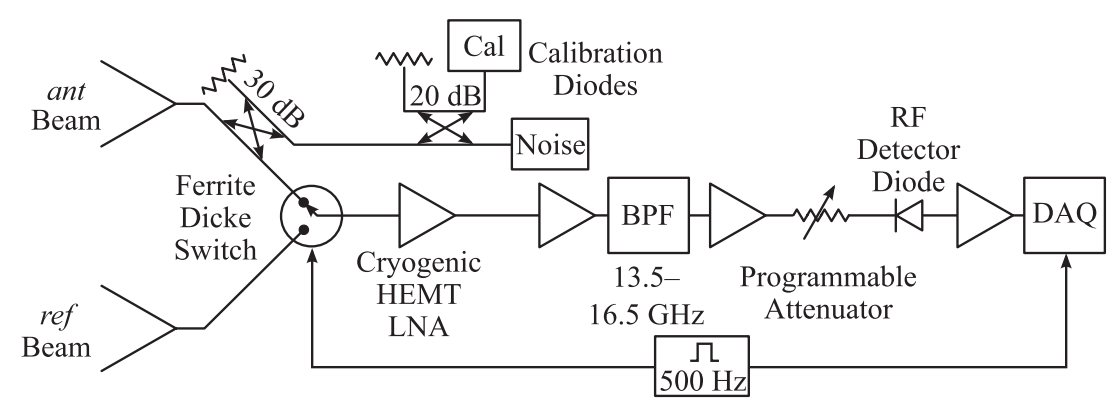

Figure 1. Block diagram of the Ku-band receiver.

Such a systematic study of radio and radio/gamma-ray population properties should allow us to address a series of longstanding questions on the physical properties of blazar jets, including the location, structure, and radiative properties of the gamma-ray emission region, and the collimation, composition, particle acceleration, and emission mechanisms in blazar jets.

In this paper, we describe in detail the $40 \mathrm{~m}$ telescope $15 \mathrm{GHz}$ monitoring program, we present results from the first two years of the program (2008 and 2009), and we derive the variability amplitude properties of the blazar population at $15 \mathrm{GHz}$. Studies of other blazar population radio and radio/gamma ray properties will be discussed in upcoming publications (e.g., W. MaxMoerbeck et al. 2011, in preparation; V. Pavlidou et al. 2011, in preparation; A. Abdo et al. 2011, in preparation; L. Fuhrmann et al. 2011, in preparation).

The remainder of this paper is organized as follows. In Section 2, we discuss the telescope and receiver and our measurement procedures. In Section 3, we discuss the method of operation. In Section 4, we discuss our sample of sources and observing strategy. In Section 5, we discuss data editing and calibration. Our results, including light curves, the derivation of variability amplitudes for the blazar population, and population studies using this analysis are presented in Section 6. We summarize and discuss our conclusions in Section 7.

\section{TELESCOPE AND RECEIVER}

\subsection{Optics}

The OVRO " $40 \mathrm{~m}$ " telescope is actually a 130 -foot-diameter $f / 0.4$ parabolic reflector with approximately $1.1 \mathrm{~mm}$ rms surface accuracy on an altitude-azimuth mount. A cooled receiver with two symmetric off-axis corrugated horn feeds is installed at the prime focus. The telescope and receiver combination produces a pair of approximately Gaussian beams (157" FWHM), separated in azimuth by $12^{\prime} .95$. We refer to these two beams, somewhat arbitrarily, as the "antenna" beam and the "reference" beam, or ant and ref. The receiver selects left-hand circular polarization, so linearly polarized sources of all orientations may be monitored in total intensity. By observing compact sources of known flux density, we find the aperture efficiency, $\eta_{A} \sim 0.25$.

This relatively low aperture efficiency is due to deliberate underillumination of the dish by the feed-for monitoring observations of a large sample of objects aiming at flux density measurements repeatable to within a few percent we must consider the tradeoff between aperture efficiency and pointing accuracy. Underillumination of the antenna increases the beamwidth and reduces susceptibility to pointing errors relative to more fully illuminating the antenna, in addition to reducing exposure to thermal noise from ground spillover. Experience has shown that we are operating at close to the optimum illumination for the most efficient use of the telescope: increasing the aperture efficiency gains little because the thermal noise is already acceptably low for observing the objects in our monitoring sample. The on-source duty cycle is about $20 \%$ - a factor of two in efficiency is lost due to Dicke switching, and the rest of the lost time is due to slewing and calibration.

When the $40 \mathrm{~m}$ telescope moves in elevation, gravity deforms its surface, changing the antenna gain and focus location. The entire feed/receiver system can be moved along the optical axis to adjust the focus. The optimum focus position as a function of elevation is measured about once per year, but has not been found to vary significantly except when the receiver has been removed and reinstalled during maintenance. Due to thermal effects, the optimum focus also varies slightly between day and night operation and with the angle between the telescope structure and the Sun.

\subsection{Receiver}

A block diagram of the receiver is shown in Figure 1. The receiver operates in the $\mathrm{Ku}$ band with a center frequency of $15.0 \mathrm{GHz}$, a $3.0 \mathrm{GHz}$ bandwidth, and a noise-equivalent reception bandwidth of $2.5 \mathrm{GHz}$. The receiver noise temperature is about $30 \mathrm{~K}$, and the typical system noise temperature including $\mathrm{CMB}$, atmospheric, and ground contributions is about $55 \mathrm{~K}$.

In order to make the most efficient use of the telescope, a Dicke-switched dual-beam system is used (e.g., Rohlfs \& Wilson 2000). A ferrite switch alternately selects between the ant and ref beams and delivers the difference between the two, which is the switched power, i.e., the difference between the power in ant beam and the power in the ref beam. We designate this power difference by $\xi$, i.e.,

$$
\xi=P_{\text {ant }}-P_{\text {ref }} \text {. }
$$

Although Dicke switching halves the time spent observing the object it is more efficient than using a single beam and scanning the telescope across the source because the integrated signal from the source is higher and hence flux densities can be measured faster, and in addition, as described below, Dicke switching removes large systematic errors.

The receiver front end consists of a cooled $(T \sim 80 \mathrm{~K})$, lowloss ferrite RF Dicke switch followed by a cryogenic $(T \sim 13 \mathrm{~K})$ HEMT low-noise amplifier. This is followed by additional roomtemperature amplifiers, a $13.5-16.5 \mathrm{GHz}$ band definition filter, and an electronically controlled attenuator used to adjust the overall gain of the receiver. The signal is detected directly using a square law detector diode. The detected signal is digitized with a 16-bit analog-to-digital converter and then recorded.

From 2007 until 2008 November, several receiver calibrations were performed. Beginning in 2008 November, approximately 
monthly calibrations were performed to monitor receiver performance. These calibrations included $Y$-factor measurements to characterize receiver temperature, sky dips to measure atmospheric optical depth and to determine the ground spillover, calibration diode effective temperature measurements, and observations of calibration sources to measure the aperture efficiency.

\subsubsection{Measurement Procedures}

In typical radiometry observations on the $40 \mathrm{~m}$ telescope we use three procedures: (1) flux density measurements, (2) measurements of a calibration noise source, and (3) pointing measurements on a nearby bright source. The receiver output voltage is integrated and digitally sampled at $1 \mathrm{kHz}$, synchronously with the $500 \mathrm{~Hz}$ Dicke-switching rate. Alternate millisecond samples are subtracted to demodulate the Dicke switching, and the results are accumulated into $1 \mathrm{~s}$ averages. In addition to the demodulated outputs, the sum of the alternate samples, i.e., the total powers in both the ant and ref beams are also recorded.

\subsubsection{Calibration Diode}

A pair of calibrated noise diodes, referred to as the noise and cal diodes, are connected to the main beam input via directional couplers to the Dicke switch. These noise diodes provide an excess noise ratio of $(31 \pm 1) \mathrm{dB}$ from 12 to $18 \mathrm{GHz}$ with stability of about $0.001 \mathrm{~dB} / \mathrm{K}$. The diodes provide two calibration levels-one of power comparable to the system temperature and one attenuated to provide power comparable to the astronomical sources we are observing. The equivalent noise temperatures of the noise and cal diodes at the receiver input are about $67 \mathrm{~K}$ and $1 \mathrm{~K}$, respectively, stable to $\ll 1 \%$.

\subsection{Pointing}

The $40 \mathrm{~m}$ telescope is equipped with encoders on the azimuth and elevation shafts and with two orthogonal tilt meters located in the teepee of the telescope in the alidade above the azimuth bearing. These four sets of readings are combined in a pointing model that generates encoder azimuth and zenith angle offsets based on the requested position on the sky. The pointing model has nine terms for the azimuth angle correction and five terms for the zenith angle correction,

$$
\begin{aligned}
\Delta \phi_{\text {model }}= & A_{1} \sin \theta+A_{2}+A_{3} \sin \phi \cos \theta \\
& +A_{4} \cos \phi \cos \theta+A_{5} \cos \theta+A_{6} \sin \phi \sin \theta \\
& +A_{7} \cos \phi \sin \theta+A_{8} \sin (4 \theta)+A_{9} T_{\mathrm{LR}} \cos \theta \\
\Delta \theta_{\text {model }}= & Z_{1}+Z_{2} \sin \theta-Z_{3} \cos \phi+Z_{4} \sin \phi+Z_{5} T_{\mathrm{AF}} .
\end{aligned}
$$

Here, $\phi$ and $\theta$ are the requested azimuth and zenith angles, $\Delta \phi_{\text {model }}$ and $\Delta \theta_{\text {model }}$ are the pointing model corrections for the azimuth and zenith angles, $A_{i}$ and $Z_{i}$ are the pointing model coefficients, and $T_{\mathrm{AF}}$ and $T_{\mathrm{LR}}$ are the aft-forward and left-right tilt meter readings.

We have found that the pointing model terms drift slowly with time. Figure 2 shows the residual offset between the pointing model and the actual requested position for 2008 and 2009. The sharp steps in the average offset correspond to adjustments in the pointing model. We adjust the pointing model two to three times per year to minimize the scatter in the offset and maintain an average offset less than about 0.5 to ensure accurate pointing. Early in 2008 and at the end of 2009, the offset approached 1', but because the scatter did not increase, there was no substantial impact on data quality.

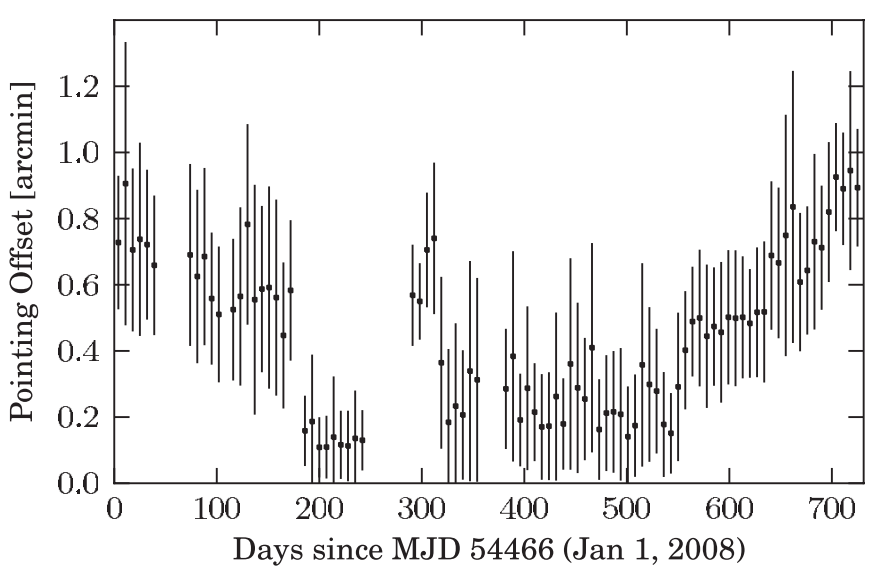

Figure 2. Residual error between the pointing model and the actual requested position, plotted in week-long bins. The plotted data and errors are the weekly means and standard deviations of the pointing offsets measured by the pointing calibrations.

In addition to the pointing model correction, at least once per hour we measure the pointing offset between a bright pointing calibrator and the model prediction. This measures the effect of wind and thermal loading. In early 2009, we determined that these pointing offsets have the accuracy we require only at separations up to about $30^{\circ}$ from the position where the pointing offset was measured. Because of this effect, after MJD 54906 (2009 March 16), care was taken when scheduling to ensure that flux density measurements were always made at separations of less than $15^{\circ}$ from the pointing offset measurement. Prior to this, no such limit was in place. We have discarded flux densities measured with a separation of more than $30^{\circ}$.

The pointing offset is measured by performing 3-point crossscans of the calibrator in both azimuth and zenith angle and fitting a fixed-width Gaussian beam profile to each axis to determine the position of the peak. A pointing offset measurement is considered invalid if its signal-to-noise ratio is less than 2 or if the offset indicates that the peak was outside the span of the cross-scan, \pm FWHM/2. Several iterations are attempted, moving the cross-scan center by up to FWHM/ 2 after each attempt, allowing offsets less than the FWHM $\left(157^{\prime \prime}\right)$ to be measured reliably.

\section{METHOD OF OBSERVING}

\subsection{Double Switching}

The observing method we used follows closely that which we developed and described in detail in Readhead et al. (1989), and also discussed in Angelakis et al. (2009). To remove the large varying total power signal and minimize the effects of the atmospheric fluctuations, ground spillover, and gain fluctuations we use a "double switching" approach, with a Dicke switch operating at $500 \mathrm{~Hz}$, and azimuth switching in which we alternate the beams on the object of interest. The advantage of double switching is that large variable signals are eliminated and the disadvantage is the loss of a factor of two in sensitivity-a factor of $\sqrt{2}$ lost through observing the object only half the time, and another factor of $\sqrt{2}$ lost through the noise introduced by subtracting off the reference field.

\subsubsection{Dicke Switching}

The most important benefit of Dicke switching is the removal of the large, slowly varying total power signal, which is made up 
of contributions from ground, atmosphere, and receiver thermal noise. Variations in the gain of the low noise amplifier cause variations in the large total power signal, and in addition the signals themselves vary slowly with time and with the position of the telescope. The resulting large variations in power limit the sensitivity of the receiving system. Ground spillover, like gain variations, contributes directly to the system noise, but the effect is difficult to quantify due to the complexity of the far sidelobes of the telescope beam. Dicke switching removes these large slowly varying signals.

A second benefit of Dicke switching is the reduction of noise due to the rapidly varying atmosphere above the telescope. With a beam separation of $12 ! 95$, and for a water vapor scale height of $1.5 \mathrm{~km}, 75 \%$ of the total mass of water vapor seen by the telescope lies in the overlapping portions of the two beams. This fraction does not change substantially with scale height, dropping only to $72 \%(70 \%)$ for a water vapor scale height of $2 \mathrm{~km}(2.5 \mathrm{~km})$. So Dicke switching reduces the effects of the varying atmosphere by about a factor of four.

A third benefit of Dicke switching is the relatively short observing time compared to the time required to scan across a radio source with a single beam. A detailed discussion of these benefits is given in Readhead et al. (1989).

\subsubsection{Beam Switching and Flux Density Measurements}

While Dicke switching does much to reduce the large error terms due to the atmosphere, the ground, and gain fluctuations in the receiver, it does not remove linear drifts in any of these quantities and the situation can be further improved by beam switching. Beam switching in azimuth is optimum because by maintaining a constant elevation we minimize changes to the atmospheric and ground spillover signals and thereby maximize their cancellation. We therefore adopt the same "double switching" technique used by Readhead et al. (1989), in which we alternate the two beams on the object of interest, and hence remove both the constant term and any linear drifts in the power from these unwanted components of the signal.

The procedure we use for measuring flux densities is identical to that described in detail in Readhead et al. (1989) so we do not repeat all the details here, but give only a summary. To begin with the ref beam is positioned on the source for $8 \mathrm{~s}$, and the power difference, $\xi_{A}$, is recorded. Then the ant beam is positioned on the source for $8 \mathrm{~s}$ and the power difference, $\xi_{B}$, is recorded. With the ant beam still on the source a second $8 \mathrm{~s}$ observation is then made and the difference, $\xi_{C}$, is recorded. Finally, the ref beam is again positioned on the source for a final $8 \mathrm{~s}$ period and the difference, $\xi_{D}$, is recorded. Thus, we spend a total of 32 s actually integrating on the source for each flux density measurement. Of course, slewing and settling times have to be allowed for at the beginning of the $A, B$, and $D$ integrations, so that the total time required for the flux density measurement is about 1 minute.

The corresponding flux density is given by

$$
S_{15}=\frac{\kappa}{4}\left(\xi_{B}+\xi_{C}-\xi_{A}-\xi_{D}\right),
$$

where $\kappa$ is the calibration factor required to turn digitizer units into $\mathrm{Jy}$, and the rms error is given by

$$
\sigma_{15}=\frac{\kappa}{4} \sqrt{\sigma_{A}^{2}+\sigma_{B}^{2}+\sigma_{C}^{2}+\sigma_{D}^{2}}
$$

The calibration factor consists of a relative calibration factor that is computed for each measurement (Section 5.2) and an absolute calibration factor (Section 5.3).
Table 1

Confusion

\begin{tabular}{lcc}
\hline \hline Flux Density Limit (mJy) & Probability & Sources Affected $^{\mathrm{a}}$ \\
\hline 100 & $8.4 \times 10^{-4}$ & 1 \\
50 & $1.9 \times 10^{-3}$ & 2 \\
20 & $5.3 \times 10^{-3}$ & 6 \\
10 & $1.2 \times 10^{-2}$ & 14 \\
\hline
\end{tabular}

Note. ${ }^{\text {a }}$ Expected number of contaminated program sources, considering a source contaminated if a confusing source is found in the source field or in either of its two reference fields.

The four measurements also contain interesting information on the stability of the instrument and, more importantly, the atmosphere, during the observations. For each flux density measurement, we therefore also compute two other quantities-one that we call the "switched power," $\psi$, given by

$$
\psi=\frac{\kappa}{4}\left(\xi_{B}+\xi_{D}-\xi_{A}-\xi_{C}\right)
$$

and the other that we call the "switched difference," $\mu$, given by

$$
\mu=\frac{\kappa}{4}\left(\xi_{C}+\xi_{D}-\xi_{A}-\xi_{B}\right) .
$$

Both $\psi$ and $\mu$ should be zero in the absence of gain or atmospheric drifts so we use these as a way of estimating such variations in our error model (Section 5.4) and to reject badly contaminated measurements (Section 5.1.5). The uncertainties in $\psi$ and $\mu$ are clearly given by Equation (5).

\subsection{Confusion}

For sources at galactic latitude $|b|>10^{\circ}$ most of the reference fields are empty, but there are some objects that are contaminated by confusion introduced by other radio sources in the field. Fortunately, since we are observing bright sources confusion is not a problem. At $15.2 \mathrm{GHz}$, Waldram et al. (2010) report a differential source count $n(S) \approx 51(S / \mathrm{Jy})^{-2.15} \mathrm{Jy}^{-1} \mathrm{sr}^{-1}$ with no deviation to a completeness limit of $5.5 \mathrm{mJy}$. Assuming that the effect of source clustering is negligible, the expected number of confusing sources detected at or above a flux density limit $S_{c}$ in either the ant or ref beam is

$$
N\left(S_{c}\right)=\int_{S_{c}}^{\infty} n(S) \Omega(S) d S,
$$

where $\Omega(S)$ is the beam solid angle with antenna gain sufficient to detect a source of flux density $S$ at the $S_{c}$ level. For a beamswitched flux density measurement, the expected number of confusing sources in the main or either reference beam is then $v=N\left(S_{c}\right)+2 N\left(S_{c} \sqrt{2}\right)$ where the confusion limit is higher in the reference beams because each is integrated only half as long as the main beam. Considering the confusing sources to be independently distributed among the observed fields via a Poisson process, the probability that a beam-switched flux density measurement includes one or more confusing sources is $1-e^{-v}$.

Table 1 shows the probability of a confusing radio source lying in the main field or either reference field of a single flux density measurement, as well as the expected number of contaminated sources in our 1158 object sample. Here, we have treated the ant and ref beams as identical 157" FWHM Gaussian beams and neglected reference field rotations with 
parallactic angle. The latter approximation is justified because we observe sources at approximately the same local sidereal time each cycle, limiting the parallactic angles at which sources are observed. Because only about $1.2 \%$ of our sources are likely to be contaminated even at $10 \mathrm{mJy}$ level ( $\sim 3 \%$ of the median flux density of sources in our sample), we may safely ignore the effects of confusion in our statistical analyses.

\section{OBSERVATIONS}

\subsection{Source Selection}

The selection of the core sample for our monitoring program was driven by three considerations. First, since we are interested in the detailed study of the radio variability properties of the blazar population and the dependence of these properties on other observables such as redshift, the sample should be sufficiently large to allow division in subsamples (e.g., in redshift or luminosity bins) with enough members to derive confidently the statistical properties in each.

Second, to allow for the evaluation of the confidence level of any correlations or variable dependencies identified in our data through Monte Carlo simulations, and the generalization of our findings to the blazar population, the sample should be well-defined statistically, using uniform and easily reproducible criteria.

Finally, one of the major goals of our monitoring program is the cross-correlation of $15 \mathrm{GHz}$ light curves with Fermi gammaray light curves. For this reason we would like our sample to include a large number of gamma-ray-loud blazars. On the other hand, we would also like to be able to address the question of why some blazars are gamma-ray-loud while other blazars, with apparently similar properties, are not. For this reason we would like our sample to be preselected - before Fermi data bias our understanding of what constitutes a likely gamma-ray-loud blazar - and, ideally, to include a comparable number of blazars which are not gamma-ray loud.

Blazars in the Candidate Gamma-Ray Blazar Survey (CGRaBS) satisfy all of the requirements above (Healey et al. 2008). CGRaBS blazars were selected from a flat-spectrum parent sample (complete to $65 \mathrm{mJy}$ flux density at $4.8 \mathrm{GHz}$ and radio spectral index $\alpha>-0.5$ where $S \propto \nu^{\alpha}$ ) by a well-defined figure-of-merit criterion based on radio spectral index, $8.4 \mathrm{GHz}$ radio flux density, and X-ray flux based on counts/s in the ROSAT All Sky Survey, to resemble blazars that were detected by the Energetic Gamma-Ray Experiment Telescope (EGRET, the precursor of Fermi-LAT). The CGRaBS sample is a total of 1625 AGNs over the whole sky outside $\mathrm{a} \pm 10^{\circ}$ band around the galactic plane. This sample was compiled before the launch of Fermi and was expected to contain a large fraction of the extragalactic sources that would be detected by Fermi-LAT.

The core sample for our monitoring program consists of the $1158 \mathrm{CGRaBS}$ sources north of declination $-20^{\circ}$. As published, our subset of the CGRaBS sample contains 812 FSRQs, 111 BL Lacs, 53 radio galaxies, and 182 objects without spectroscopic identification. In our analysis we use redshifts from the CGRaBS publication, which covered $81 \%$ of the sample (100\% of FSRQs, $49 \%$ of BL Lacs). Recent spectroscopy has improved the completeness of the sample to 886 FSRQs, 122 BL Lacs, 60 radio galaxies, and 88 objects without spectroscopic identification, with redshifts now available for $87.5 \%$ of the sample (100\% of FSRQs; $53 \%$ of BL Lacs). The median $15 \mathrm{GHz}$ flux density for sources in our sample ranged from about $20 \mathrm{mJy}$ to $30 \mathrm{Jy}$ with a median of $325 \mathrm{mJy}$ during the observation period described in this paper.

In 1LAC, 709 AGNs were associated with 1FGL sources (Abdo et al. 2010b). Although continued improvements in evaluating the probability of radio counterpart identification have caused some source associations to vary in estimated significance and continued optical observations have improved the completeness of the typing and redshifts, we adopt here the identifications published in the 1LAC (Abdo et al. 2010b). These identifications include 291 CGRaBS sources (221 of our subset) that were associated with sources detected in gammarays by Fermi (Abdo et al. 2010b). Of these, 263 (199 of our subset) were considered "clean" associations, meaning only one source was associated with the gamma-ray source and the association probability was greater than $80 \%$. CGRaBS sources made up $44 \%$ of the clean associations in the first-year Fermi AGN catalog. This number is thus far smaller than anticipated; in the 11 month 1LAC sample only $16 \%$ of the CGRaBS sources were detected and a large number of blazars not in CGRaBS have been detected. This suggests that the CGRaBS (EGRET-like) blazar sample is substantially different from that seen in the early Fermi mission. This finding represents a unique opportunity to investigate why gamma-ray activity is found only in certain blazars, and for this reason we retain in our monitoring program all of the blazars in our original core sample even if they have not yet been detected by the LAT. However, in order to optimize the potential for studies of the cross-correlation between radio and gamma-ray light curves, we have since added (and we continue to add) to our monitoring program all new Fermi-LAT blazars north of $-20^{\circ}$ declination that are not CGRaBS members.

Several bright, stable non-blazar sources are included in our program to provide flux density calibration and to monitor instrumental variability. These are 3C 48, 3C 161, 3C 274, 3C 286 , and DR 21 . In addition to the stable sources, a number of bright sources are used to calibrate pointing. These sources need not exhibit stable flux density, but need be brighter than about $100 \mathrm{mJy}$ to permit pointing offsets to be measured reliably.

In addition to the core samples of blazars discussed above we have added further small samples of objects to our biweekly monitoring program, including (1) any objects not already included in our sample that are being studied in the F-GAMMA or VERITAS programs, (2) a variety of galactic objects, such as microquasars and cataclysmic variables, and (3) a few bright radio galaxies that show interesting jet properties. We are continually adding sources of interest to our monitoring sample, so that by now the sample comprises over 1500 objects that are monitored twice weekly.

\subsection{Scheduling}

The large number of sources being observed requires the development of strategies to optimize the use of the telescope and minimize the effect of known systematic errors. The principal systematic errors we try to minimize are gain variations, atmospheric optical depth variations, and pointing errors. To achieve this optimization while minimizing slew times and dead times between observations requires careful planning. Due to the size of our sample the scheduling must be automated.

Schedules are arranged to ensure that sources are observed between zenith angles of $20^{\circ}$ and $60^{\circ}$ whenever possible. This is done for a number of reasons:

1. the figure of the telescope was set for maximum gain in this elevation range; 
2. at zenith angles less than about $20^{\circ}$ the telescope has to move rapidly to track an object and pointing accuracy can be degraded;

3. at zenith angles greater than about $60^{\circ}$ ground spillover increases significantly with decreasing elevation;

4. it is desirable to minimize the variation in atmospheric optical depth on our sources so as to minimize this particular source of error; and

5. we try to minimize telescope slew times by observing to the south and east in a limited elevation range.

In the scheme we have developed, the sky is divided into 192 cells, each with a diameter $\lesssim 20^{\circ}$, using the HEALPix mesh with $N_{\text {side }}=4$ (Górski et al. 2005). Each source is assigned to a cell. From the sources in each cell, a pointing calibrator is selected using the following criteria, applied in order: (1) if there is a flux calibrator in the region, this source is selected; (2) if one or more sources in the region have a flux density larger than $500 \mathrm{mJy}$, the one which minimizes the average angular distance to all the sources in that region is selected; and (3) the source with the largest flux density in the region is selected. For these flux density comparisons, the median flux density of the source during the previous year's observations is used.

Sources within the region are ordered to minimize slew time, using a direct search to find the optimal order for regions with fewer than nine sources and simulated annealing for regions with nine or more sources. A second optimization step determines the order in which the regions are scheduled using a heuristic algorithm in which regions are observed within a fixed zenith angle range and regions to the south have priority. The total sample is observed in three days.

Prior to MJD 54906 (2009 March 16), a scheduling algorithm was used which did not enforce an angular separation limit between pointing calibrators and subsequent flux density measurements.

\section{DATA EDITING AND CALIBRATION}

\subsection{Data Editing and Flagging}

Editing and removal of corrupted data is performed using both automated and manual filters.

\subsubsection{Wind, Sun, Moon, and Zenith Angle Cuts}

Under high winds there is a systematic reduction in observed flux densities due to mispointing and poor tracking. Observations when the wind speed exceeds $6.7 \mathrm{~m} \mathrm{~s}^{-1}(15 \mathrm{mph})$ are discarded. To protect the telescope a "wind watchdog" program stows the telescope pointing at the zenith when winds exceed steady $8.9 \mathrm{~m} \mathrm{~s}^{-1}(20 \mathrm{mph})$ or gusts above $13.4 \mathrm{~m} \mathrm{~s}^{-1}(30 \mathrm{mph})$. The telescope remains stowed until the wind speed has remained below these thresholds for $1 \mathrm{hr}$.

Observations at zenith angles $<20^{\circ}$ are discarded because the telescope is unable to track fast enough in azimuth to match the sidereal rate near zenith. The scheduling algorithm avoids scheduling sources for observation at these zenith angles, so few observations are lost. Observations at solar or lunar elongations less than $10^{\circ}$ are also discarded. The scheduler does not avoid these areas of the sky so a small number of observations are lost.

\subsubsection{Pointing and Calibration Failures}

An observation is rejected if a pointing offset was not obtained within the prior $4800 \mathrm{~s}$ or if the pointing offset measurement immediately preceding the observation failed.
Occasional scheduling errors resulted in observations without adequately measured pointing offsets. These observations are discarded.

An observation is rejected if fewer than two reliable calibration procedures using the cal diode were successfully executed within a $2 \mathrm{hr}$ interval centered on the time of the observation, or if the largest and smallest cal diode measurements within that interval differ by more than $10 \%$.

\subsubsection{Saturation or Total Power Anomalies}

The total power varies depending on the attenuator setting, receiver gain fluctuations, atmospheric conditions, and the observed zenith angle. Observations that indicate saturation or other total power anomalies are rejected. Heavy cloud cover or precipitation often causes large fluctuations in total power. Such periods are identified by inspection of the total power time series and manually discarded. Negative flux density measurements are indicated by the $95 \%$ upper limits on these values.

\subsubsection{Measured Uncertainty}

We reject flux density measurements with anomalously large measured uncertainties, $\sigma_{15}$ (Equation (5)). However, a straightforward cut at a fixed value or a fixed multiple of the expected thermal uncertainty introduces a bias against larger flux densities. This occurs because there are contributions to the measured error that are proportional to the flux density of the target radio source, such as telescope tracking errors. We therefore apply a flux density-dependent threshold and discard flux density measurements for which

$$
\sigma_{15}>\zeta \sqrt{1+\left(\rho \cdot S_{15}\right)^{2}}
$$

The optimal values of $\zeta=0.0208$ and $\rho=0.2$ were estimated from the data to eliminate as many unreliable measurements as possible while minimizing flux density bias. About $2 \%$ of the data is eliminated by this filter.

\subsubsection{Switched Difference}

We also use the switched difference $\mu$, defined by Equation (7), to determine whether flux density measurements might be contaminated by systematic errors. The expected value of $\mu$ is 0 , provided that the ground spillover and atmospheric noise in the ant and ref beams are identical. Pointing and tracking errors again give flux density-dependent contributions to $\mu$, so to avoid bias against brighter radio sources we flag points where

$$
\left|\frac{\mu}{\sigma_{15}}\right|>\beta \cdot \frac{\left(\mu_{0}+\rho_{s} \cdot S_{15}\right)}{\sqrt{1+\left(\rho_{t} \cdot S_{15}\right)^{2}}} .
$$

Again the optimum values of the parameters $\left(\beta=5, \mu_{0}=\right.$ $1.148, \rho_{s}=0.0682$, and $\rho_{t}=0.0243$ ) are determined from the data. This procedure gives consistent results across calibration epochs and it discards about $2 \%$ of flux densities, with comparable fractions dropped from each epoch.

\subsection{Relative Calibration}

To correct for slow gain fluctuations of the receiver, we first divide each flux density measurement by a calibration factor measured using the small noise diode cal. A measurement of the strength of the cal diode is made after each pointing observation and no less than once per hour. Because gain fluctuations are slow, the calibration factor is averaged over a $2 \mathrm{hr}$ window, 
Table 2

Absolute Calibration Epochs

\begin{tabular}{lcccc}
\hline \hline Epoch & MJD & Date & MJD & Date \\
\hline 1 & 54466 & 2008 Jan 1 & 54753 & 2008 Oct 14 \\
2 & 54753 & 2008 Oct 14 & 54763 & 2008 Oct 24 \\
3 & 54763 & 2008 Oct 24 & 55197 & 2010 Jan 1 \\
\hline
\end{tabular}

centered on the time of the flux density measurement. If there are fewer than two good measurements of the strength of the cal diode in that window then the flux density observation is discarded.

Due to gravitational deformation of the telescope structure and the increase in atmospheric attenuation with airmass, the effective antenna gain varies substantially with zenith angle. We model this variation with a polynomial gain curve and scale flux density measurements to remove the effect. The gain curve is measured by observing bright calibrator sources across the full range of zenith angles. Day-to-day changes in atmospheric opacity are found to vary with $<1 \% \mathrm{rms}$, so these variations are accounted for within our error model and no correction is applied.

Additionally, the optimal axial focus position varies with zenith angle, as well as solar zenith angle and elongation. During observations, the focus position is set using a polynomial model of the zenith angle variation and a correction is applied during calibration using a more complete model that accounts for solar zenith angle and elongation.

The combined effect of these corrections is a factor, $\kappa_{\text {rel }}$, that is computed for each flux density measurement.

\subsection{Absolute Calibration}

We divide our observation period into epochs characterized by a consistent ratio between the calibration diode and feed horn inputs to the receiver. This ratio might change if, for example, the signal path is disconnected and reconnected for maintenance, resulting in a slight change in loss along one path. Within a single epoch, the ratio of the calibration diode signal to a stable astronomical source should therefore be constant. Table 2 lists the epochs we have used in our analysis. Absolute calibration is applied to each epoch separately.

For each epoch, a calibration factor is determined from regular observations of the primary calibrator, 3C 286. We adopt the spectral model and coefficients from Baars et al. (1977). At our $15 \mathrm{GHz}$ center frequency, this yields $3.44 \mathrm{Jy}$, with a quoted absolute uncertainty of about $5 \%$. The calibration factor for epoch $i, \kappa_{i}$, is the ratio of the adopted flux density for the calibrator to the weighted mean of the observations:

$$
\kappa_{i}=\frac{3.44 \mathrm{Jy}}{\left(\sum S_{15}^{\prime} \cdot \sigma_{15}^{\prime}{ }^{-2}\right) /\left(\sum \sigma_{15}^{\prime}{ }^{-2}\right)},
$$

where $S_{15}^{\prime}$ and $\sigma_{15}^{\prime}$ denote the flux densities for the calibrator with only the relative calibration applied.

The total calibration factor for a flux density measurement in Equation (4) is then $\kappa=\kappa_{\text {rel }} \cdot \kappa_{i}$ and reflects both relative and absolute calibration. Comparing our calibrated flux densities for 3C 48, 3C 161, and DR 21 with the Baars et al. (1977) values, we find an absolute scale error of $(-0.8 \pm 4.1) \%$. Cross-checks of our calibration against $14.6 \mathrm{GHz}$ observations of a number of common sources observed with the Effelsberg $100 \mathrm{~m}$ telescope through the F-GAMMA project confirm the overall accuracy of our flux density scale.
Table 3

Error Model Parameter Values

\begin{tabular}{lcll}
\hline \hline Parameter & Pointing Calibrator & Early $^{\mathrm{a}}$ & Late $^{\mathrm{a}}$ \\
\hline$\epsilon$ & 0.0057 & 0.0200 & 0.0135 \\
$\eta$ & 3.173 & 3.173 & 3.173 \\
\hline
\end{tabular}

Note. " The "early" model applies prior to MJD 54906 (2009 March 16).

\subsection{Uncertainties in Individual Flux Density Measurements}

In a perfect observing system with no sources of systematic error the uncertainties in the flux density measurements would be given by the thermal noise in each observation. In practice there are many sources of systematic error, including the effects of weather and the atmosphere, mispointing due to wind, and focus errors. Many of these are correctly identified and accounted for in the automatic and manual editing described in Section 5.1. However, even after flux density measurements affected by these problems are filtered out there remain many observations that are significantly affected by systematic errors. Such systematic errors can lead to significant errors in the measurement that are not reflected in the thermal noise of the observation and can give rise to bad flux density measurements with small thermal errors. This leads to "outliers" in the light curves, i.e., points which do not lie close to the level determined from interpolation of adjacent observations and which have small errors. The task of identifying and eliminating or allowing for the wide variety of systematic errors leading to such outliers is challenging and time-consuming. Great care must be taken not to assume that the behavior of the source is known, and hence to eliminate a real and potentially extremely interesting flux density variation.

We first apply an error model to determine the uncertainty of each flux density measurement:

$$
\sigma_{\text {total }}^{2}=\sigma_{15}^{2}+\left(\epsilon \cdot S_{15}\right)^{2}+(\eta \cdot \psi)^{2},
$$

which is an extension of the model described in Angelakis et al. (2009). The first term represents the measured scatter during the flux density measurement. This includes thermal noise, rapid atmospheric fluctuations, and other random errors. The second term adds an uncertainty proportional to the flux density of the source. This term allows for pointing and tracking errors, variations in atmospheric opacity, and other effects that have a multiplicative effect on the measured flux density. In the third term $\psi$ is the switched power, defined by Equation (6). This term takes account of systematic effects that cause the A-B segment of the flux density measurement to differ from the C-D segment, such as a pointing offset between the $A$ and D segments, or some rapidly varying weather conditions.

The error model is defined by the two parameters, $\epsilon$ and $\eta$, whose values must be determined from the observations. Because $\epsilon$ describes the error contribution due to pointing errors, its value depends on whether a source is used as a pointing calibrator. Furthermore, for non-pointing sources, $\epsilon$ is found to differ between the scheduling algorithms used before and after MJD 54906 (2009 March 16). The parameter, $\eta$, is found to be adequately described by a single value for all sources and all epochs. The adopted values are given in Table 3.

For pointing sources, both $\epsilon$ and $\eta$ were estimated simultaneously using the stable flux calibrators 3C 286, 3C 48, 3C 161 , and DR 21. Due to systematic errors, these sources and other stable-flux density calibrators show long-term variations 


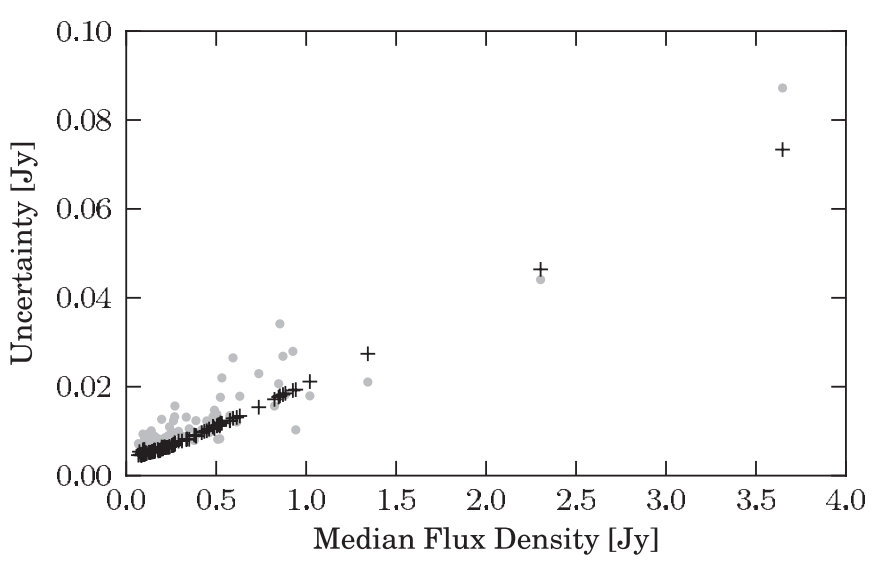

Figure 3. Residual standard deviation (gray points) and fitted $\epsilon$-only error model values (black crosses) for ordinary sources in the early (MJD < 54906) period. The fit in the late period is similar. A single high-flux density data point was omitted to limit the scale.

of $1 \%-2 \%$ so we fitted a seventh-order polynomial to remove this trend from each source, then computed the residual standard deviation, median flux density, the rms, and mean $\psi$ for each source, then used these to fit the error model parameters.

To determine the error model parameter $\epsilon$ for ordinary sources, we selected 100 sources that exhibited little variation or slow, low-amplitude variations in flux density, between the start of our program and MJD 55048 (2009 August 5). This interval was split into two periods, "early" and "late," at MJD 54906 and this procedure was separately applied to each period. For each light curve, we fitted and removed a second-order polynomial trend, then iteratively removed outlier data points with residuals greater than three standard deviations. We repeated the fitting and outlier removal until no further outliers were removed and we discarded any source with fewer than 10 remaining data points (retaining 94 and 88 sources in the early and late periods, respectively). From the surviving points in each light curve, we computed the median and the rms flux densities, and the standard deviation of the residuals. We then fitted Equation (12) to these data, omitting the $\eta$ term. The data and the error model results for the early period are shown in Figure 3 . We then adopted the same value of $\eta$ for these sources as was determined for pointing sources.

\subsubsection{Long-term Trends in 3C 286, 3C 274, and DR 21}

After carrying out the above editing and calibration steps we returned to the residual $1 \%-2 \%$ long-term ( $\sim 6$ month) variations in the light curves for stable-flux-density calibration sources. We chose 3C 286, 3C 274, and DR 21 for this study because they are well known to be stable on timescales of many years. The fractional variations in flux density of these objects are shown in Figure 4 and are clearly correlated, indicating the presence of an unidentified source of multiplicative systematic error. For each of these sources, we removed $2 \sigma$ outliers in a 100 day sliding window and normalized the resulting data by the median flux density. We then combined the data for all three sources and fitted a cubic spline to the result.

We apply the corresponding correction to all light curves in our program by dividing each flux density by the value of this spline. Figure 5 shows the residuals for the three fitted sources after dividing out the spline fit. The $1 \%$ residual variation that remains is the level of systematic uncertainty after correction for this long-term trend.

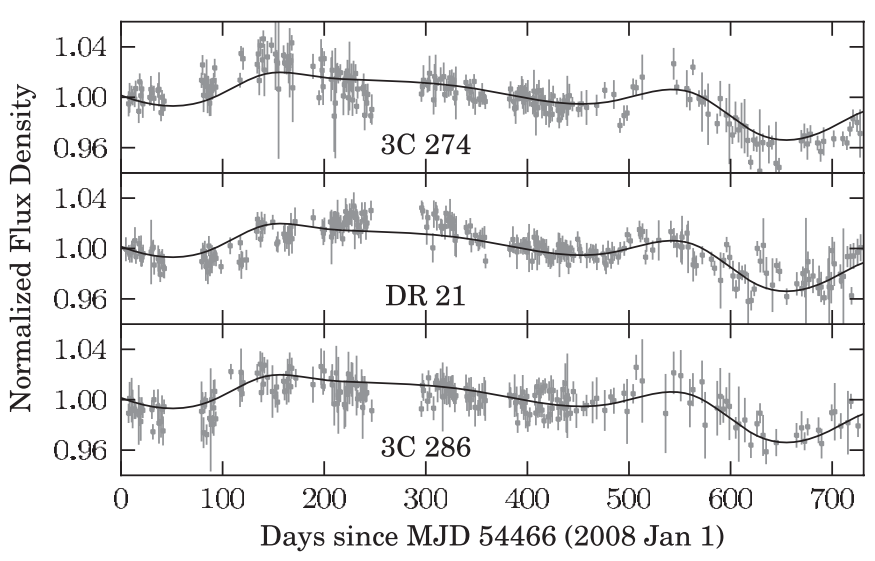

Figure 4. Normalized flux densities for 3C 274 (top), DR 21 (center), and 3C 286 (bottom) after outlier removal. Each light curve is normalized by its median. The black line in each plot is the spline fit to the combined data.

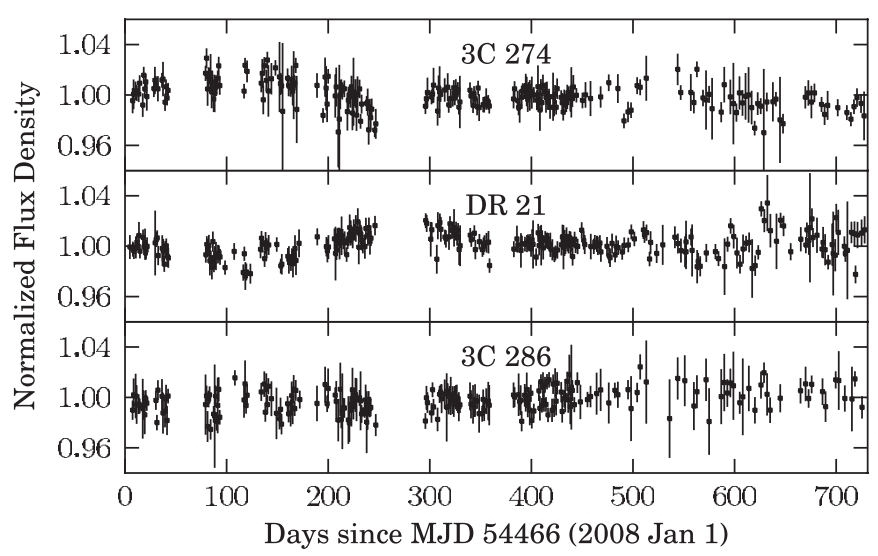

Figure 5. Normalized flux densities for 3C 274 (top), DR 21 (center), and 3C 286 (bottom) after dividing by the spline fit to remove long-term systematic trends.

\subsection{Scaling of the Non-thermal Error}

The reported error for each flux measurement has two qualitatively different components as described in Section 5.4. The first component is directly obtained during the flux measurement and it represents random errors such as thermal noise and rapid atmospheric fluctuations, while the second is introduced to take into account other, flux-density-dependent effects. This error model requires the determination of constant factors, which we have called $\epsilon$ and $\eta$, and which have been assumed to be source independent. However, many sources exhibit coherent long-term variations with scatter about those clearly smaller than what would be expected as a result of the quoted errors. This is a direct indication that in certain cases the simple assumption of source-independent $\epsilon$ and $\eta$ resulted in overestimated errors.

To correct these constant scale factors on a source-by-source basis, we have used cubic spline fits and required the $\chi^{2}$ per degree of freedom to be one for the residuals. Due to the large number of sources and the requirement of a uniform and consistent method for all the sources, an automatic method was developed for this procedure. For each source we can in principle use a range of number of polynomial sections to construct a spline fit. We construct a spline fit for each possible number of polynomial sections. ${ }^{12}$ An outlier rejection filter which uses a cubic spline fit with a small number of knots is used to fit the

12 We use the MATLAB Spline Toolbox function spap2, which automatically selects the positions of the knots for the spline. 

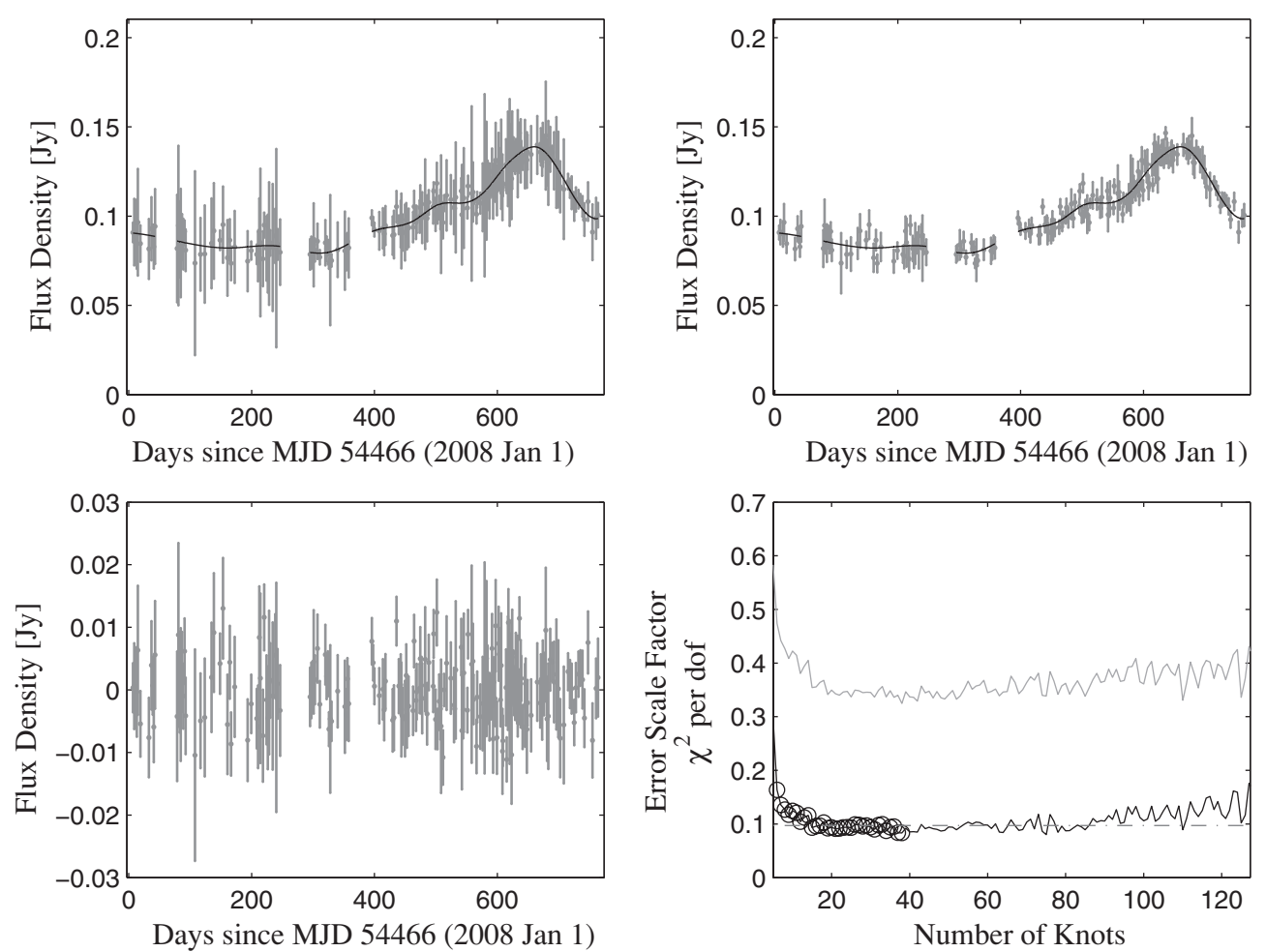

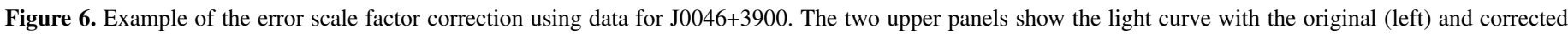

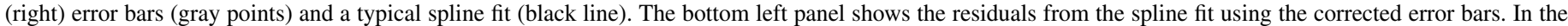

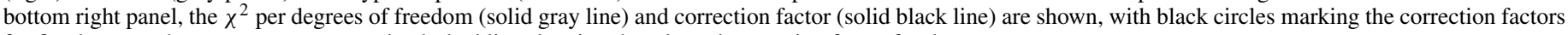
for fits that pass the acceptance tests, and a dashed line showing the adopted correction factor for the source.

light curve. Points with absolute residuals in the largest $5 \%$ are not used for the following stage of the fitting procedure. Not all the fits are acceptable, as some cases will have correlated residuals or a large departure from normality. Acceptable fits are selected by using two statistical tests: Lilliefors' test for normality (Lilliefors 1967) and the runs test for randomness (e.g., Wall \& Jenkins 2003). ${ }^{13}$ Only the fits for which both null hypotheses cannot be rejected at the $10^{-3}$ level are considered acceptable. For each acceptable fit, a scale factor that makes the $\chi^{2}$ per degree of freedom equal to one is calculated. Among the scale factors for all the acceptable fits, the median scale factor is selected as the final correction. The value of the scale factor is not very sensitive to the exact number of polynomial sections. A typical example of the behavior of the scale factor is shown in Figure 6.

We have thus only rescaled the non-thermal part of the errors (the $S_{15}$ and $\psi$ terms in Equation (12)), and only for those sources for which the resulting correction factor was smaller than one (i.e., the rescaling would result in smaller errors). The latter choice was made for two reasons. First, a correction factor larger than one simply indicates that the spline fit cannot provide an adequate description of the data. This may result from a light curve more variable than can be fit by spline with a given number of knots, so such a correction could mask real variability. Only the reverse is cause for concern-when the spline fit is too good a fit, given the quoted errors. Second, this choice ensures a smooth transition between scaled and non-scaled errors, as the transition point (correction factor equal to one) is equivalent to no error scaling.

${ }^{13}$ We have used the implementation of both tests that are part of the MATLAB Statistics Toolbox.

\section{RESULTS AND ANALYSIS}

Properties of our program sample and calibrator sources are presented in Table 4.

\subsection{Monitoring Program Statistics}

Our target cadence was two flux density measurements per source per week, or 208 measurements per source in the first two years of the program. The number of successful observations for each source is listed in Table 4. Our resulting average effective cadence for CGRaBS sources is about 128 measurements per source in the first two years of the program. The efficiency compared to our nominal cadence is $62 \%$.

\subsection{Light Curves}

Light curves for the CGRaBS program sources and calibrators are shown in Figures 7.1-7.1163. Table 5 lists the filtered and calibrated $15 \mathrm{GHz}$ flux density measurements that result from the procedure described above. Regular updates to the data set, including data for sources outside the core sample released in this paper, are available from the program Web site. ${ }^{14}$

\subsection{Source Variability}

In this section, we discuss the variability amplitude observed in each source in our sample. The variability properties of our sources in the time and frequency domains, as quantified by measures such as the power spectrum and autocorrelation function, and correlation with gamma-ray data to identify and measure time lags, will be discussed in detail in a forthcoming publication (W. Max-Moerbeck et al. 2011, in preparation).

\footnotetext{
14 http://www.astro.caltech.edu/ovroblazars
} 

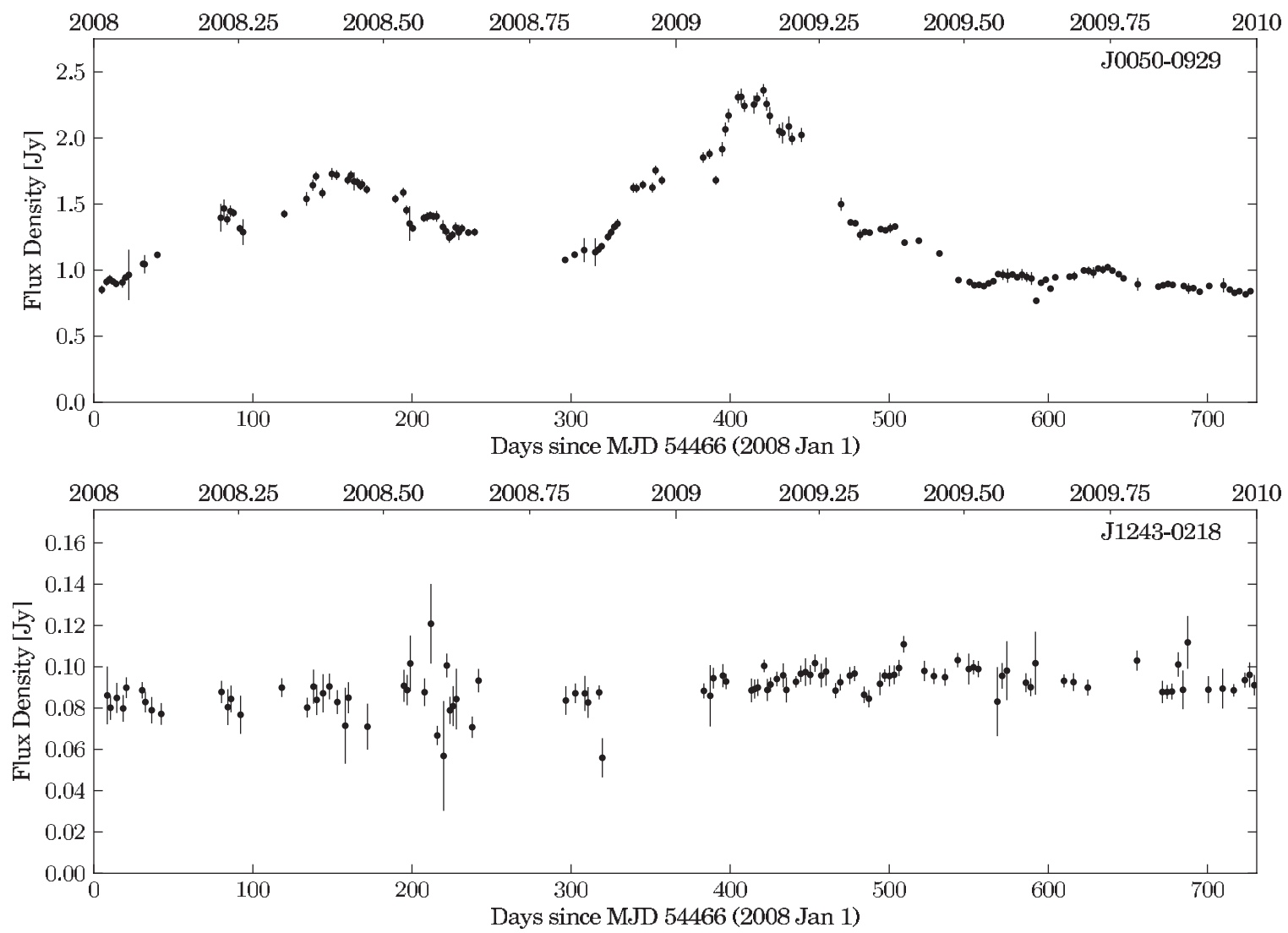

Figure 7. $15 \mathrm{GHz}$ light curves for calibrators and CGRaBS program sources.

(The complete figure set (1163 images) is available in the online journal.)

Table 4

Program Source Data

\begin{tabular}{|c|c|c|c|c|c|c|c|c|c|c|}
\hline Name & R.A. & Decl. & $z^{\mathrm{a}}$ & $\begin{array}{l}\text { Optical } \\
\text { Class }^{\mathrm{a}}\end{array}$ & $\begin{array}{c}\mathrm{Num}^{\mathrm{b}} \\
\mathrm{Obs}\end{array}$ & 1 LAC? & Flag $^{\mathrm{d}}$ & $\chi^{2} ?^{e}$ & $\begin{array}{l}S_{0}{ }^{\mathrm{f}} \\
(\mathrm{Jy})\end{array}$ & $\begin{array}{l}\bar{m}^{\mathrm{f}} \\
(\%)\end{array}$ \\
\hline $3 \mathrm{C} 48$ & $01^{\mathrm{h}} 37^{\mathrm{m}} 41^{\mathrm{s}} 30$ & $33^{\circ} 09^{\prime} 35^{\prime \prime} 1$ & $\ldots$ & $\ldots$ & 274 & $\mathrm{~N}$ & 5 & V & $1.804 \pm 0.002$ & $1.7 \pm 0.1$ \\
\hline $3 \mathrm{C} 161$ & $06^{\mathrm{h}} 27^{\mathrm{m}} 10^{\mathrm{s}} .12$ & $-05^{\circ} 53^{\prime} 05^{\prime \prime} .2$ & $\ldots$ & $\ldots$ & 182 & $\mathrm{~N}$ & 5 & V & $2.085 \pm 0.003$ & $1.8 \pm 0.1$ \\
\hline $3 \mathrm{C} 274$ & $12^{\mathrm{h}} 30^{\mathrm{m}} 49^{\mathrm{s}} .42$ & $12^{\circ} 23^{\prime} 28^{\prime \prime} .0$ & $\ldots$ & .. & 243 & $\mathrm{Y}$ & 4 & $\mathrm{C}$ & $26.335 \pm 0.022$ & $0.9 \pm 0.1$ \\
\hline $3 \mathrm{C} 286$ & $13^{\mathrm{h}} 31^{\mathrm{m}} 08.29$ & $30^{\circ} 30^{\prime} 33^{\prime \prime} .0$ & $\ldots$ & $\ldots$ & 224 & $\mathrm{~N}$ & 4 & $\mathrm{C}$ & $3.438 \pm 0.003$ & $0.6 \pm 0.1$ \\
\hline DR21 & $20^{\mathrm{h}} 39^{\mathrm{m}} 01^{\mathrm{s}} \cdot 20$ & $42^{\circ} 19^{\prime} 32^{\prime \prime} .9$ & $\ldots$ & $\ldots$ & 276 & $\mathrm{~N}$ & 4 & $\mathrm{C}$ & $19.020 \pm 0.011$ & $0.7 \pm 0.1$ \\
\hline J0001+1914 & $00^{\mathrm{h}} 01^{\mathrm{m}} 08^{\mathrm{s}} .62$ & $19^{\circ} 14^{\prime} 33^{\prime \prime} .8$ & 3.100 & FSRQ & 160 & $\mathrm{~N}$ & 0 & V & $0.284 \pm 0.003$ & $11.1_{-0.7}^{+0.8}$ \\
\hline $\mathrm{J} 0001-1551$ & $00^{\mathrm{h}} 01^{\mathrm{m}} 05^{\mathrm{s}} .33$ & $-15^{\circ} 51^{\prime} 07^{\prime \prime} .1$ & 2.044 & FSRQ & 152 & $\mathrm{~N}$ & 0 & V & $0.213 \pm 0.002$ & $8.7_{-0.6}^{+0.7}$ \\
\hline $\mathrm{J} 0003+2129$ & $00^{\mathrm{h}} 03^{\mathrm{m}} 19.35$ & $21^{\circ} 29^{\prime} 44^{\prime \prime} 4$ & 0.450 & AGN & 162 & $\mathrm{~N}$ & 0 & V & $0.087 \pm 0.001$ & $7.9_{-0.7}^{-0.6}$ \\
\hline $\mathrm{J} 0004+2019$ & $00^{\mathrm{h}} 04^{\mathrm{m}} 35^{\mathrm{s}} .76$ & $20^{\circ} 19^{\prime} 42^{\prime \prime} .2$ & 0.677 & BLL & 169 & $\mathrm{~N}$ & 0 & V & $0.324 \pm 0.003$ & $12.0_{-0.7}^{+0.8}$ \\
\hline $\mathrm{J} 0004+4615$ & $00^{\mathrm{h}} 04^{\mathrm{m}} 16^{\mathrm{s}} .13$ & $46^{\circ} 15^{\prime} 18^{\prime \prime} .0$ & 1.810 & FSRQ & 146 & $\mathrm{~N}$ & 0 & V & $0.180 \pm 0.006$ & $39.5_{-2.7}^{+3.0}$ \\
\hline J0004-1148 & $00^{\mathrm{h}} 04^{\mathrm{m}} 04^{\mathrm{s}} .92$ & $-11^{\circ} 48^{\prime} 58^{\prime \prime} .4$ & $\ldots$ & BLL & 98 & $\mathrm{~N}$ & 0 & $\mathrm{~V}$ & $0.716 \pm 0.012$ & $16.1_{-1.2}^{+1.3}$ \\
\hline
\end{tabular}

Notes.

${ }^{\text {a }}$ For the CGRaBS sample, redshift and optical classifications are repeated here from Healey et al. (2008) for convenience.

${ }^{b}$ The number of observations that survived data editing and were used in our variability analysis.

${ }^{\mathrm{c}}$ Is the source associated with a gamma-ray source in the Fermi 1LAC catalog (Abdo et al. 2010b).

${ }^{\mathrm{d}}$ Variability analysis flag. A value of 0 indicates a non-zero intrinsic modulation is found; 1 indicates the source is non-variable; 2 indicates insufficient observations for variability analysis; 3 indicates flux density too faint for variability analysis; 4 indicates the source was a calibrator used in the spline fit to remove long-term trends; 5 indicates the source was a calibrator not used in the spline fit.

${ }^{\mathrm{e}}$ Result of $3 \sigma \chi^{2}$ test for variability. A C (V) indicates the source is (is not) consistent with a constant flux density.

${ }^{\mathrm{f}}$ Quoted errors are $1 \sigma$ uncertainties. Values for non-variable sources indicate $3 \sigma$ upper limits and no uncertainties are quoted for $\bar{m}$ or $S_{0}$.

(This table is available in its entirety in a machine-readable form in the online journal. A portion is shown here for guidance regarding its form and content.)

The questions of the variability amplitude of a source and the confidence with which this can be measured are complex ones and have been traditionally addressed using a variety of measures and tests, such as the variability index (e.g., Aller et al. 1992); the fluctuation index (e.g., Aller et al. 2003); the modulation index (e.g., Kraus et al. 2003); the fractional variability amplitude (e.g., Edelson et al. 2002; Soldi et al. $2008)$; and $\chi^{2}$ tests of a null hypothesis of non-variability. 
Table 5

$15 \mathrm{GHz}$ Flux Densities

\begin{tabular}{ccc}
\hline \hline Source & MJD & Flux Density (Jy) \\
\hline J0001-1551 & 54471.051377 & $0.244 \pm 0.008$ \\
J0001-1551 & 54474.042836 & $0.232 \pm 0.007$ \\
J0001-1551 & 54478.032303 & $0.221 \pm 0.009$ \\
J0001-1551 & 54480.026840 & $0.238 \pm 0.011$ \\
J0001-1551 & 54484.015903 & $0.229 \pm 0.008$ \\
\hline
\end{tabular}

(This table is available in its entirety in a machine-readable form in the online journal. A portion is shown here for guidance regarding its form and content.)

Each of these tools provides different insights to the variability properties of sources and is sensitive to different uncertainties, biases, and systematic errors. For example, the variability index, defined as the peak-to-trough amplitude change of the flux, is a measure of the amplitude of the variability of a source

$$
V=\frac{\left(S_{\max }-\sigma_{\max }\right)-\left(S_{\min }+\sigma_{\min }\right)}{\left(S_{\max }-\sigma_{\max }\right)+\left(S_{\min }+\sigma_{\min }\right)},
$$

where $S_{\max }$ and $S_{\min }$ are the highest and lowest measured flux densities, respectively, and $\sigma_{\max }$ and $\sigma_{\min }$ are the uncertainties in these measurements. Although the definition is constructed to account for the effect of measurement uncertainties, the quantity is well defined only when variability is significantly greater than measurement errors, and it can yield negative values for sources with low signal-to-noise ratios or little intrinsic variability. In addition, it is very sensitive to outliers and is not robust against random Gaussian excursions from the mean. Such excursions are to be expected for sources that are regularly monitored over long periods of time: even non-variable sources are likely to have at least one pair of $2 \sigma$ high and low measurements after being observed more than 100 times, as is the case for most sources in our sample.

An associated measure of variability amplitude is the modulation index, defined as the standard deviation of the flux density measurements in units of the mean measured flux density,

$$
m_{\text {data }}=\frac{\sqrt{\frac{1}{N} \sum_{i=1}^{N}\left(S_{i}-\frac{1}{N} \sum_{i=1}^{N} S_{i}\right)^{2}}}{\frac{1}{N} \sum_{i=1}^{N} S_{i}} .
$$

The modulation index has the advantage that it is always non-negative and more robust against outliers. However, it still represents a convolution of intrinsic source variation and observational uncertainties: a large modulation index could be indicative of either a strongly variable source or a faint source with high uncertainties in individual flux density measurements. For this reason, the correct interpretation of results on the modulation index requires that measurement errors and the uncertainty in $m$ due to the finite number of flux density measurements be properly accounted for.

One method that has been widely used to evaluate the information encoded in variability measures is to evaluate each measure for a set of constant-flux-density calibrators, which are known to have a flux density constant in time and have been observed with the same instrument over the same periods of time. The value of the variability measure obtained for the calibrators is then used as a threshold value, so that any source with variability measure equal to or lower than that of the calibrators is considered consistent with being nonvariable. However, a variability measure value higher than that of the calibrators is a necessary but not sufficient condition for establishing variability: calibrators are generally bright sources, with relative flux density measurement uncertainties typically lower than the majority of monitored sources; additionally, variability measures are affected by the sampling frequency, which is not necessarily the same for all monitored sources and the calibrators.

Alternatively, the significance of variability in a given source can be established through tests (such as a $\chi^{2}$ test) evaluating the consistency of the obtained set of measurements with the hypothesis that the source was constant over the observation interval. However, such tests provide very little information on sources for which statistically significant variability cannot be established, as they cannot distinguish between intrinsically non-variable sources and sources that could be intrinsically variable but inadequately observed for their variability to be revealed.

Here, we propose a new index for characterizing source variability: the intrinsic modulation index $\bar{m}$, which is the intrinsic standard deviation of the distribution of source flux densities in time, $\sigma_{0}$, measured in units of the intrinsic source mean flux density, $S_{0}$. Here, the term "intrinsic" is used to denote flux densities and variations as would be observed with perfectly uniform sampling of adequate cadence and zero observational error:

$$
\bar{m}=\frac{\sigma_{0}}{S_{0}}
$$

In this way, $\bar{m}$ is a measure of the true amplitude of variations in the source, rather than a convolution of true variability, observational uncertainties, and effects of finite sampling. Observational uncertainties and finite sampling will, of course, affect the accuracy with which $\bar{m}$ can be measured. The purpose of the analysis described in this section is to derive a best estimate of $\bar{m}$, as well as an estimate of the uncertainty in our measurement of this quantity. For sources with $\bar{m}$ within $3 \sigma$ from zero, the $3 \sigma$ upper limit on $\bar{m}$ will be evaluated.

\subsubsection{A Likelihood Analysis to Obtain the Intrinsic Modulation Index}

For the purposes of our analysis, we will assume that the "true" flux densities for each AGN are normally distributed, with mean $S_{0}$, standard deviation $\sigma_{0}$, and intrinsic modulation index $\bar{m}=\sigma_{0} / S_{0}$. We have $N$ measurements of the flux density, $S_{j}$, each of which has an associated observational uncertainty, also assumed Gaussian, $\sigma_{j}$.

Let us assume that at a moment of observation, a source has a "true" flux density $S_{t}$. The probability density to observe a value near $S_{j}$ if the observational uncertainty is $\sigma_{j}$ is

$$
p\left(S_{t}, S_{j}, \sigma_{j}\right)=\frac{1}{\sigma_{j} \sqrt{2 \pi}} \exp \left[-\frac{\left(S_{t}-S_{j}\right)^{2}}{2 \sigma_{j}^{2}}\right]
$$

In addition, the probability density that the true source flux density at one of the moments of observation is near $S_{t}$ if the source flux densities are distributed normally with mean $S_{0}$ and standard deviation $\sigma_{0}$ is

$$
p\left(S_{t}, S_{0}, \sigma_{0}\right)=\frac{1}{\sigma_{0} \sqrt{2 \pi}} \exp \left[-\frac{\left(S_{t}-S_{0}\right)^{2}}{2 \sigma_{0}^{2}}\right]
$$




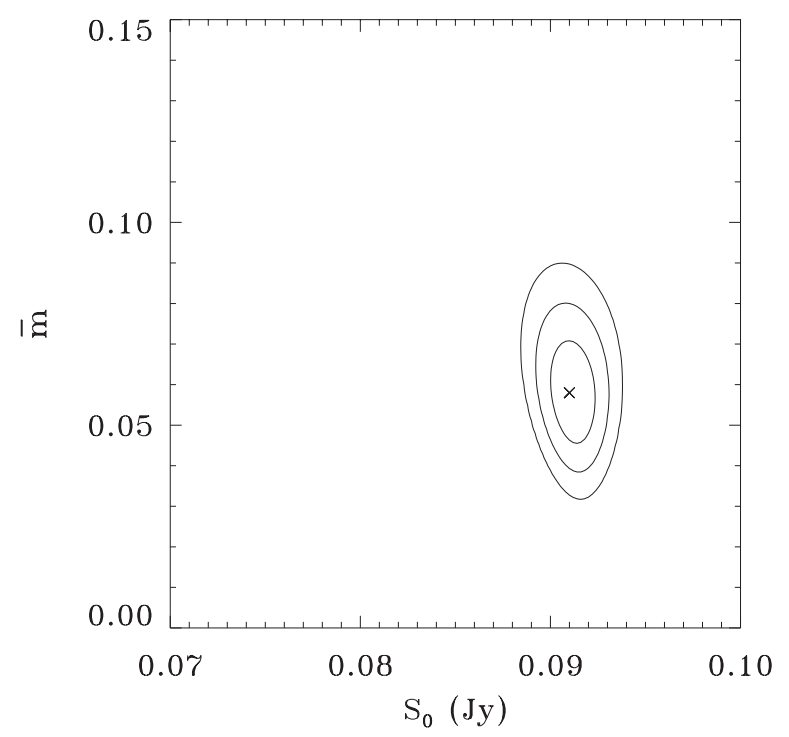

Figure 8. $1 \sigma, 2 \sigma$, and $3 \sigma$ contours of the joint likelihood $\mathcal{L}\left(S_{0}, \bar{m}\right)$ for blazar J1243-0218.

Therefore, the likelihood of observing one flux density $S_{j}$ with uncertainty $\sigma_{j}$ from the particular source is

$$
\ell_{j}=\int_{\text {all } S_{t}} d S_{t} \frac{\exp \left[-\frac{\left(S_{t}-S_{j}\right)^{2}}{2 \sigma_{j}^{2}}\right]}{\sigma_{j} \sqrt{2 \pi}} \frac{\exp \left[-\frac{\left(S_{t}-S_{0}\right)^{2}}{2 \sigma_{0}^{2}}\right]}{\sigma_{o} \sqrt{2 \pi}},
$$

which amounts to calculating the probability to observe $S_{j}$ through any possible true flux density value $S_{t}$. If the limits of integration above are taken to be from $S_{t}=-\infty$ to $S_{t}=\infty$ then the integral has an analytic solution (see, e.g., Venters \& Pavlidou 2007):

$$
\ell_{j}=\frac{1}{\sqrt{2 \pi\left(\sigma_{0}^{2}+\sigma_{j}^{2}\right)}} \exp \left[-\frac{\left(S_{j}-S_{0}\right)^{2}}{2\left(\sigma_{j}^{2}+\sigma_{0}^{2}\right)}\right] .
$$

The likelihood for $N$ observations $\left(S_{j}, \sigma_{j}\right)$ for $j=1, \ldots, N$ is

$$
\begin{aligned}
\mathcal{L}\left(S_{0}, \sigma_{0}\right)=\prod_{j=1}^{N} \ell_{j}= & \left(\prod_{j=1}^{N} \frac{1}{\sqrt{2 \pi\left(\sigma_{0}^{2}+\sigma_{j}^{2}\right)}}\right) \\
& \times \exp \left[-\frac{1}{2} \sum_{j=1}^{N} \frac{\left(S_{j}-S_{0}\right)^{2}}{\sigma_{j}^{2}+\sigma_{0}^{2}}\right] .
\end{aligned}
$$

The intrinsic standard deviation $\sigma_{0}$ can be eliminated in favor of the intrinsic modulation index,

$$
\sigma_{0}=\bar{m} S_{0}
$$

so that

$$
\begin{aligned}
\mathcal{L}\left(S_{0}, \bar{m}\right)= & S_{0}\left(\prod_{j=1}^{N} \frac{1}{\sqrt{2 \pi\left(\bar{m}^{2} S_{0}^{2}+\sigma_{j}^{2}\right)}}\right) \\
& \times \exp \left[-\frac{1}{2} \sum_{j=1}^{N} \frac{\left(S_{j}-S_{0}\right)^{2}}{\sigma_{j}^{2}+\bar{m}^{2} S_{0}^{2}}\right] .
\end{aligned}
$$

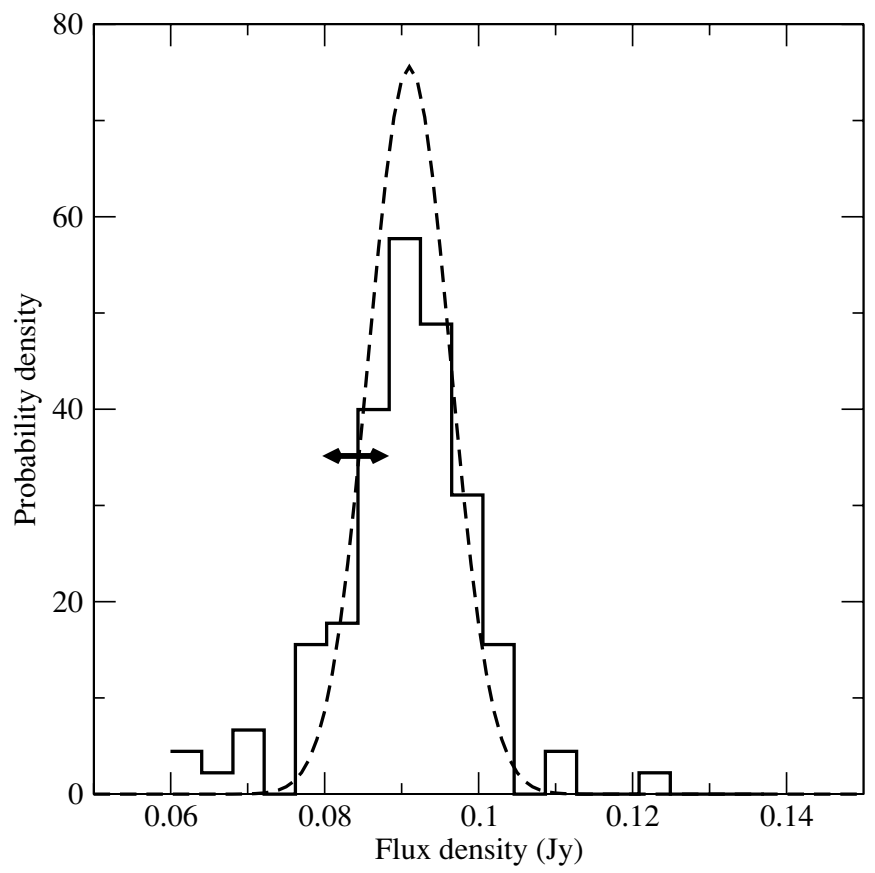

Figure 9. Maximum-likelihood Gaussian model for the flux density distribution (dashed line), plotted over the histogram of measured flux densities (solid line) for blazar J1243-0218. The arrow indicates the size of the typical measurement uncertainty.

This likelihood is symmetric about $\bar{m}=0$, as $\bar{m}$ only enters through its square. For this reason, this formalism can guarantee non-negative intrinsic modulation indices without loss of information.

Maximizing the joint likelihood $\mathcal{L}\left(S_{0}, \bar{m}\right)$, we can derive maximum-likelihood estimates of $S_{0}$ and $m$. Isolikelihood contours containing $68.26 \%, 95.45 \%$, and $99.73 \%$ of the total volume under the joint likelihood surface define the $1 \sigma, 2 \sigma$, and $3 \sigma$ contours, respectively (see Figure 8 for an example in the case of J1243-0218, whose light curve is shown in Figure 7). The maximum-likelihood Gaussian for the distribution of flux densities for the same object is compared to the histogram of measurements in Figure 9. Note that the maximum-likelihood Gaussian is narrower than the histogram; this behavior is expected, as the histogram is a representation of measurements sampling the underlying distribution with finite error. The typical magnitude of the latter for the particular source is shown in Figure 9 with the blue arrows, and it is indeed comparable with the difference in width between the maximum-likelihood Gaussian and the histogram.

To derive the most likely value of $\bar{m}$ and the associated uncertainties regardless of the true value of $S_{0}$, we integrate $S_{0}$ out of $\mathcal{L}\left(S_{0}, \bar{m}\right)$, and obtain the marginalized likelihood as a function of only $\bar{m}$ :

$$
\begin{array}{r}
\mathcal{L}(\bar{m})=\int_{\text {all }_{0}} d S_{0} S_{0}\left\{\left(\prod_{j=1}^{N} \frac{1}{\sqrt{2 \pi\left(\bar{m}^{2} S_{0}^{2}+\sigma_{j}^{2}\right)}}\right)\right. \\
\left.\times \exp \left[-\frac{1}{2} \sum_{j=1}^{N} \frac{\left(S_{j}-S_{0}\right)^{2}}{\sigma_{j}^{2}+\bar{m}^{2} S_{0}^{2}}\right]\right\} .
\end{array}
$$

Then, the value of $\bar{m}$ that maximizes the marginalized likelihood is our best estimate of it, and the $1 \sigma$ uncertainty on the modulation index can be found by locating the isolikelihood 


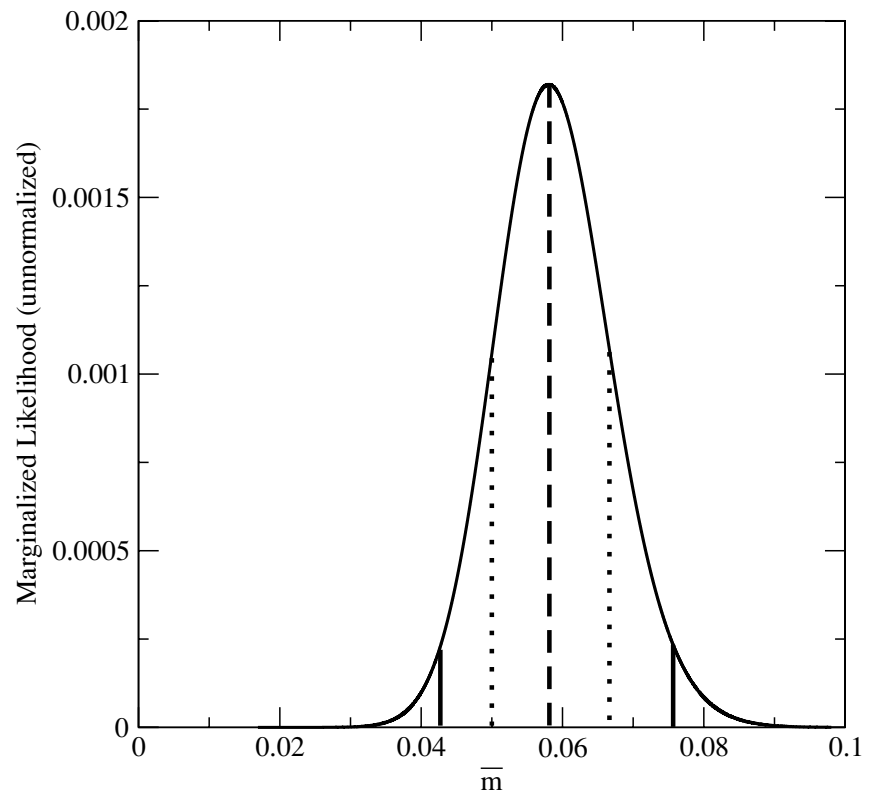

Figure 10. Marginalized likelihood $\mathcal{L}(\bar{m})$ for J1243-0218 (solid curve). Dashed vertical line: best-estimate $\bar{m}$; dotted vertical lines: $1 \sigma \bar{m}$ range; solid vertical lines: $2 \sigma \bar{m}$ range.

$\bar{m}$-values $\bar{m}_{1}$ and $\bar{m}_{2}$ for which

$$
\mathcal{L}\left(\bar{m}_{1}\right)=\mathcal{L}\left(\bar{m}_{2}\right)
$$

and

$$
\frac{\int_{\bar{m}_{1}}^{\bar{m}_{2}} \mathcal{L}(\bar{m}) d \bar{m}}{\int_{0}^{\infty} \mathcal{L}(\bar{m}) d \bar{m}}=0.6826 .
$$

Note that the upper and lower $1 \sigma$ errors are not generally symmetric in our formalism. Similarly, $2 \sigma$ and $3 \sigma$ ranges can be derived by substituting the right-hand side of Equation (25) by 0.9545 and 0.9973 , respectively. The marginalized likelihood, best-estimate $\bar{m}$, and the $1 \sigma$ and $2 \sigma \bar{m}$ ranges for blazar $\mathrm{J} 1243-0218$ are shown in Figure 10.

If the maximum-likelihood $\bar{m}$ is less than $3 \sigma$ away from $\bar{m}=0$, we consider that statistically significant variability cannot be established. In these cases, we calculate the $3 \sigma$ upper limit on $\bar{m}$, which is defined as the value $\bar{m}_{3}$ for which

$$
\frac{\int_{0}^{\bar{m}} \mathcal{L}(\bar{m}) d \bar{m}}{\int_{0}^{\infty} \mathcal{L}(\bar{m}) d \bar{m}}=0.9973 .
$$

The use of the intrinsic modulation index and the likelihood analysis we have employed to estimate it have the advantage of offering a way to obtain information about the intrinsic variability of the source, deconvolved from observational errors in individual flux density measurements and the effects of finite sampling, while providing strictly defined $1 \sigma, 2 \sigma$, and $3 \sigma$ uncertainties for our estimate of $\bar{m}$ (essential when conducting population studies), and upper limits for $\bar{m}$ when variability cannot be established at $\mathrm{a} \geqslant 3 \sigma$ confidence. However, our choice carries its own caveats.

1. Model-dependence of the likelihood analysis. A functional form has to be assumed for the intrinsic distribution of flux densities (here we have assumed it to be Gaussian), resulting in a loss of generality. The validity of this assumption can be tested by comparing the maximum-likelihood intrinsic flux density distribution to the histogram of measured flux densities, to evaluate whether the maximum-likelihood flux density distribution is a reasonable description of the data (modulo observational uncertainties). This is indeed the case for many, although not all, of our sources. An example of a source well described by the maximum-likelihood flux density distribution is shown in Figure 9. Other sources however show bimodality, and the distribution of measured flux densities in these cases could be better described by, for example, a double Gaussian. An extended likelihood analysis that does explicitly account for bimodality and calculates not only the intrinsic modulation index but also duty cycles and flaring-to-quiescent flux density ratios will be presented in an upcoming publication. For the purposes of this work we have confirmed that, even when a single Gaussian is not an adequate description of the flux density distribution, the intrinsic modulation index $\bar{m}$ is well correlated, within uncertainties, with the modulation index $m_{\text {data }}$ derived from the data (Equation (14)), which, although contaminated by observational uncertainties, is completely model-independent (see the next section).

2. Assumption of unbiased sampling. Our analysis assumed that the flux density values we have not sampled are not correlated with each other. This assumption is poor in the case of lengthy outages, as well as for increased cadence for any single epoch. In our analysis we have disregarded the additional data taken during epochs of increased cadence for specific objects (during, for example, campaigns to constrain intra-day variability).

3. Leakage of probability density to negative flux densities. In certain cases, extending the integration over intrinsic flux densities from $-\infty$ to $\infty$ (in Equation (18)) to simplify the mathematical manipulations leads to unacceptable leakage of probability density to unphysical domain of negative true flux densities. This approximation is adopted for numerical efficiency, since in this case the likelihood can be expressed analytically without the need to perform multi-dimensional integrals for every object in our large sample. For most objects in our sample the leakage to the negative flux density domain is negligible. The error introduced in this way becomes important only for very dim AGN (because the peak of the $S_{t}$ distribution is very close to zero) or very variable AGN (because of very long tails in the $S_{t}$ distribution). None of the CGRaBS sources in our sample are dim enough for the first effect to be a problem, and very few are variable enough: for $\bar{m} \sim 0.5$, about $3 \%$ of the "true flux density" probability density leaks to negative values, with the problem becoming more severe for more variable sources; only four CGRaBS sources have $\bar{m} \geqslant 0.5$.

\subsubsection{Variability Analysis-Results}

Table 4 includes our measured values for $\bar{m}, S_{0}$, and their $1 \sigma$ errors. In Figure 11, we plot the intrinsic modulation index $\bar{m}$ and associated $1 \sigma$ uncertainty against the intrinsic, maximumlikelihood average flux density, $S_{0}$, for all our CGRaBS and calibrator sources. The error bar on $S_{0}$ corresponds to the $1 \sigma$ uncertainty in mean flux density, calculated from the joint likelihood (Equation (22)) marginalized over $\bar{m}$. CGRaBS sources are shown as black or magenta points or blue triangles for upper limits, while calibrators are shown as green points.

Variability could only be established at the $3 \sigma$ confidence level or higher for 1139 out of 1158 CGRaBS blazars in our sample. For this study, we considered only sources for which at least three flux densities were measured, a positive mean 


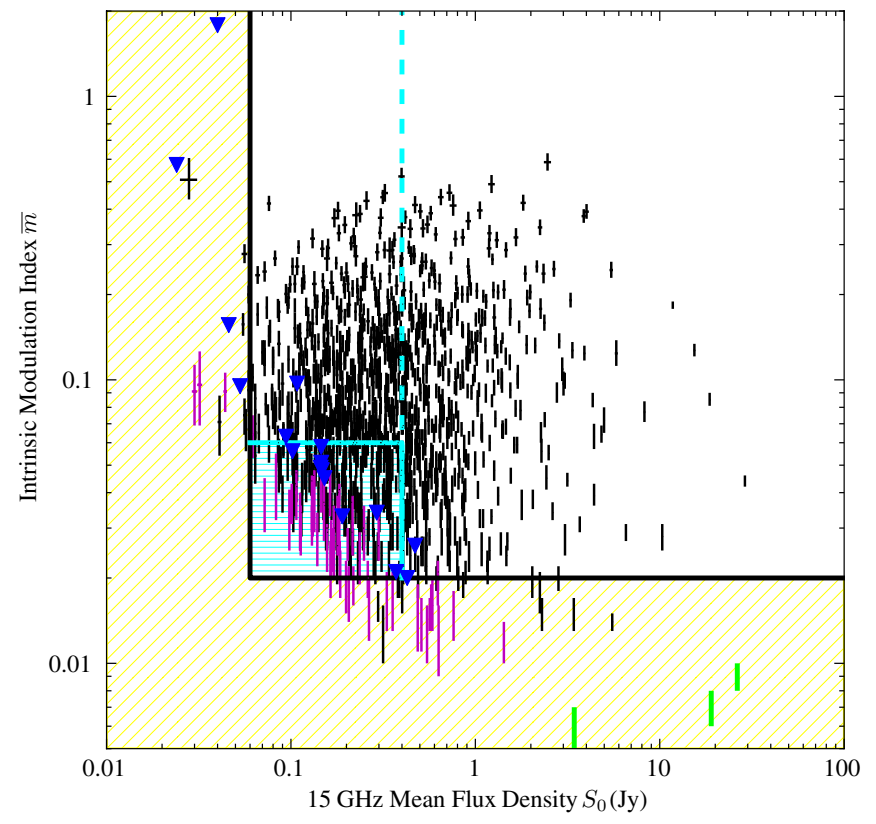

Figure 11. Intrinsic modulation index $\bar{m}$ and associated $1 \sigma$ uncertainty, plotted against intrinsic maximum-likelihood average flux density, $S_{0}$, for all sources in the program which have enough (more than 3) acceptable, non-negative flux density measurements. Black points: CGRaBS sources found to be variable with $3 \sigma$ confidence by $\chi^{2}$ test; magenta points: CGRaBS sources found consistent with non-variable by $\chi^{2}$ test; green points: calibrators $3 \mathrm{C} 286$, DR 21, and 3C 274; blue triangles: $3 \sigma$ upper limits for CGRaBS sources for which variability could not be established at $\geqslant 3 \sigma$ confidence level. The error bar on $S_{0}$ corresponds to the $1 \sigma$ uncertainty in mean flux density, calculated from the joint likelihood (Equation (22)) marginalized over $\bar{m}$. Data, except for upper limits, outside the yellow and cyan shaded areas are used in the population studies of Section 6.3.3.

flux density $\geqslant 2 \sigma$ from zero was found, and at least $90 \%$ of the individual flux density measurements were $\geqslant 2 \sigma$ from zero. These criteria excluded two sources $(\mathrm{J} 1310+3233$ and J1436-1846). For the other 17 sources we have calculated $3 \sigma$ upper limits for $\bar{m}$. We plot these upper limits with blue triangles.

Calibration sources 3C 286, DR 21, and 3C 274 are shown in green. Although these sources are the least variable (as expected) of all sources in which variability can be established and a non-zero $\bar{m}$ can be measured, $\bar{m}$ for these sources is finite and measurable. This means that some residual long-term variability remains in our calibrators beyond what can be justified by statistical errors alone. This could conceivably result from true calibrator source variation, but more likely reflects incomplete removal of small-amplitude calibration trends. Because $\bar{m}<$ $1 \%$ for these three sources, we quote a systematic uncertainty $\Delta \bar{m}_{\text {syst }}=0.01$ for the values of the intrinsic modulation index we produce through our analysis.

To ensure that our population studies are not affected by this residual systematic variability, in all analyses discussed in Section 6.3 .3 only sources with $\bar{m} \geqslant 0.02$ will be used, so that we remain comfortably above this $1 \%$ systematic uncertainty limit. In addition, for sources with $S_{0} \leqslant 60 \mathrm{mJy}$, the number of sources for which variability can be established is of the same order as the number of sources for which we could only measure an upper limit, and these upper limits are very weak and non-constraining. For this reason, we also exclude from our population studies of Section 6.3.3 all sources below $S_{0}=60 \mathrm{mJy}$. The part of the parameter space excluded due to these two criteria is shown in Figure 11 as the yellow shaded area bounded by the solid black lines.

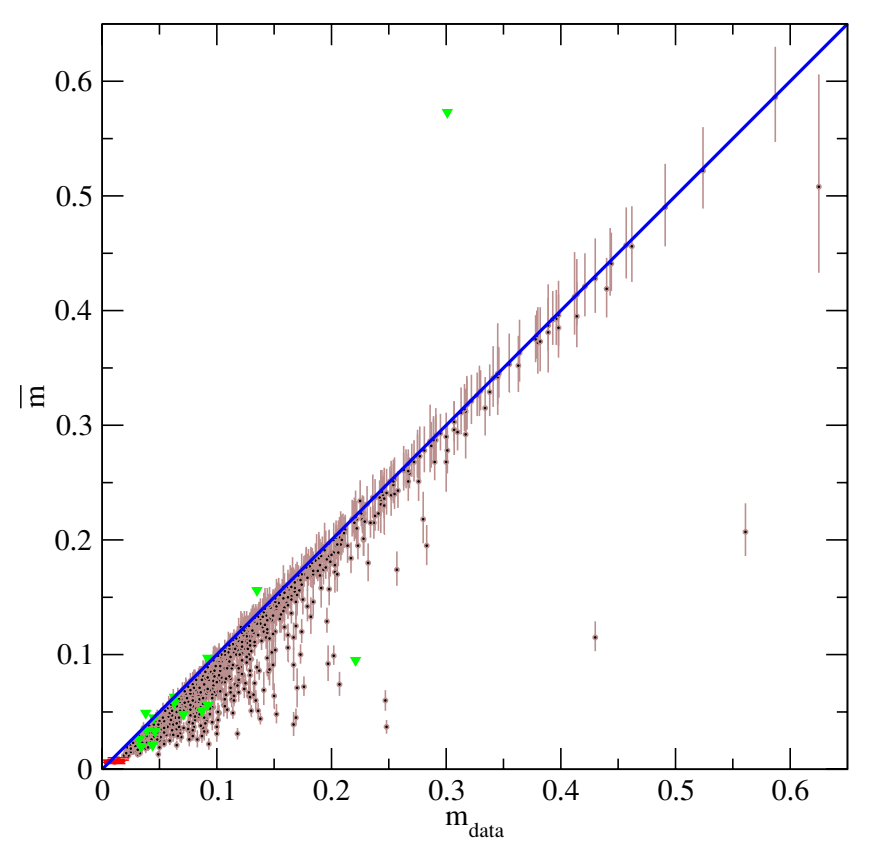

Figure 12. Intrinsic modulation index $\bar{m}$ and associated $1 \sigma$ uncertainty, plotted against the "raw" modulation index, $m_{\text {data }}$, of Equation (14) as black points with brown error bars. The $\bar{m}=m_{\text {data }}$ line is shown in blue. Green triangles are the $3 \sigma$ upper limits of sources for which variability could not be established. Calibrators 3C 286, 3C 274, and DR 21 are plotted in red.

For $S_{0} \geqslant 0.4 \mathrm{Jy}$, no obvious correlation between flux density and modulation index is apparent, and no CGRaBS sources exist with upper limits above our cut of $\bar{m}=2 \%$. However, for sources with $S_{0}<0.4 \mathrm{Jy}$, there is an absence of points in the lower-left corner of the allowed parameter space defined by the thick solid lines: for faint sources, we can only confidently establish variability if that variability is strong enough. The effect disappears for variability amplitudes greater than about $6 \%$. In addition, there are only two CGRaBS with upper limits higher than $6 \%$ for sources brighter than $60 \mathrm{mJy}(\mathrm{J} 0722+3722$ and $\mathrm{J} 0807+5117),<0.5 \%$ of the 452 sources measured in this region of parameter space. We conclude that we are able to measure variability at the level of $6 \%$ or higher for virtually all (>99\% of) sources brighter than $60 \mathrm{mJy}$.

To ensure that our population studies are not affected by our decreased efficiency in measuring variability in sources with $60 \mathrm{mJy} \leqslant S_{0} \leqslant 0.4 \mathrm{Jy}$ and $2 \% \leqslant \bar{m} \leqslant 6 \%$, we will also exclude this part of the $\left(S_{0}, \bar{m}\right)$ parameter space from our analysis in Section 6.3.3. The part of the parameter space excluded due to these criteria is shown in Figure 11 as the cyan shaded area.

For comparison, we also computed the $\chi^{2}$-per degrees of freedom for each source and tested whether we could reject the hypothesis of a constant flux density at the $3 \sigma$ level. The result of this test for each source is shown in Table 4. Because of the long-term residual trend described in Section 5.4.1, we added $1 \%$ of each flux density in quadrature to the reported uncertainty when computing $\chi^{2}$. Of the 1139 CGRaBS sources for which we calculated the intrinsic modulation index, 51 $(4.5 \%)$ are found to be non-variable (i.e., we cannot reject the hypothesis of constant flux) with $>3 \sigma$ confidence. These are plotted as magenta points in Figure 11. All but one of these lie within the low-flux density and low-variability regions we have excluded from our population studies. The one such source not excluded, J2148+0211, is very near both the flux density and intrinsic modulation index cut lines. Of the 17 sources for which we report $\bar{m}$ upper limits, 15 are judged 


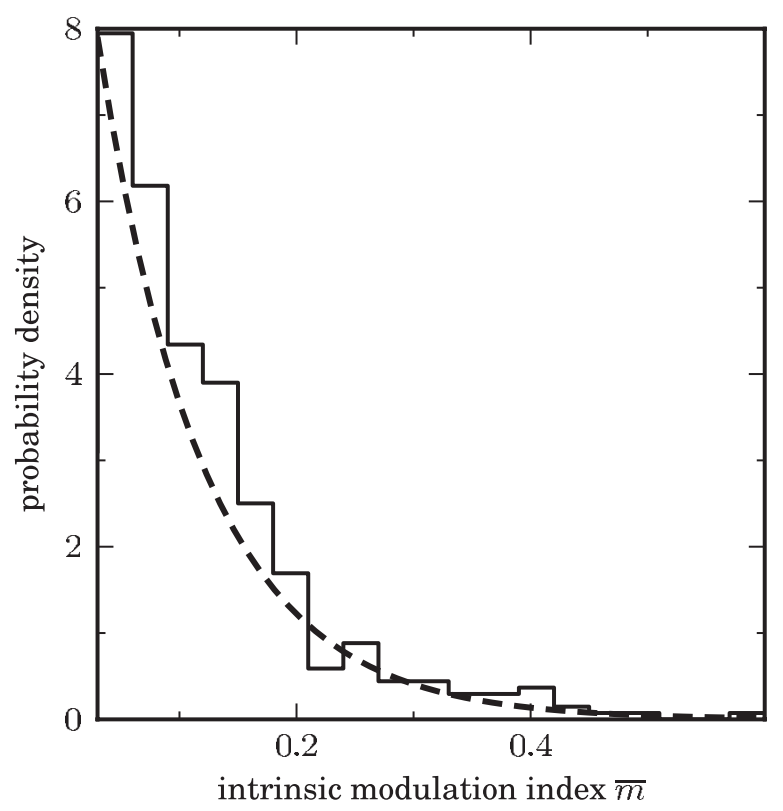

Figure 13. Histogram of maximum-likelihood intrinsic modulation indices $\bar{m}$, for the 453 CGRaBS blazars with $S_{0}>400 \mathrm{mJy}$, normalized as a probability density that integrates to unity. The dashed line represents an exponential distribution with $\langle\bar{m}\rangle=0.091$.

non-variable by the $\chi^{2}$ test. The two others are dim sources with a single outlier $(\mathrm{J} 1613+4223)$ or very few measurements (J1954+6153), which led to large uncertainties in the estimate for $\bar{m}$ and resulted in an upper limit. Calibrator sources 3C 286 , DR 21, and 3C 274 are found to be non-variable while 3C 48 and 3C 161 (which were not used to fit the long-term calibration trend) are found to be variable by the $\chi^{2}$ test, probably due to imperfect removal of the long-term calibration trend. Our estimates of $\bar{m}$ for both these calibrators are below our $2 \%$ intrinsic modulation cut level. We conclude that our analysis is generally consistent with the $\chi^{2}$ test for identifying significant variability and that our data cuts for our population studies conservatively exclude the regions of parameter space where disagreements occur.

In Figure 12 we plot the intrinsic modulation index $\bar{m}$ and associated $1 \sigma$ uncertainty against the "raw" modulation index $m_{\text {data }}$ of Equation (14). The $\bar{m}=m_{\text {data }}$ line is shown in blue. Green triangles are the $3 \sigma$ upper limits of sources for which variability could not be established. Calibrators 3C 286, DR 21, and 3C 274 are plotted in red. Since apparent variability due to the finite accuracy with which individual flux densities can be measured has been corrected out of $\bar{m}$, the expectation is that deviations from the $\bar{m}=m_{\text {data }}$ will be more pronounced for sources that are not intrinsically very variable (so that the scatter in the flux density measurements is appreciably affected, and even dominated, by measurement error). In addition, deviations are expected to be below the line, as $\bar{m}$ should be smaller than $m_{\text {data }}$. Both these expectations are verified by Figure 11 . Note that upper limits need not satisfy this criterion, as the "true" value of the modulation index can take any value below the limit. Upper limits above the blue line are weak, indicating that the reason variability could not be established is the poor sampling or quality of the data, and not necessarily a low intrinsic variation in the source flux density.

For the 453 CGRaBS objects which have $S_{0}>400 \mathrm{mJy}$ and for which variability can be established, we plot, in Figure 13, a histogram of their intrinsic modulation indices $\bar{m}$ normalized so that the vertical axis has units of probability density. The dashed line represents an exponential distribution of mean $\langle\bar{m}\rangle=0.091$ which, as we can see, is an excellent description of the data. Motivated by this plot, we will be using the monoparametric exponential family of distributions:

$$
f(m) d m=\frac{1}{m_{0}} \exp \left[-\frac{m}{m_{0}}\right] d m
$$

with mean $m_{0}$ and variance $m_{0}^{2}$, to characterize various subsamples of our blazar sample.

\subsubsection{Variability Analysis-Population Studies-Formalism}

We now turn our attention to whether the intrinsic variability amplitude at $15 \mathrm{GHz}$, as quantified by $\bar{m}$, correlates with the physical properties of the sources in our sample. To this end, we will determine the distribution of intrinsic variability indices $\bar{m}$ for various subsets of our monitoring sample, and we will examine whether the various subsets are consistent with being drawn from the same distribution.

We will do so using again a likelihood analysis. We will assume that the distribution of $\bar{m}$ in any subset is an exponential distribution of the form given in Equation (27). Since distributions of this family are uniquely described by the value of the mean, $m_{0}$, our aim is to determine $m_{0}$, or rather the probability distribution of possible $m_{0}$ values, in any specific subset.

The likelihood of a single observation of a modulation index $\bar{m}_{i}$ of Gaussian uncertainty $\sigma_{i}$ drawn from an exponential distribution of mean $m_{0}$ is

$$
\begin{aligned}
\ell_{i}= & \int_{\bar{m}=0}^{\infty} d \bar{m} \frac{1}{m_{0}} \exp \left(-\frac{\bar{m}}{m_{0}}\right) \frac{1}{\sigma_{i} \sqrt{2 \pi}} \exp \left[-\frac{\left(\bar{m}-\bar{m}_{i}\right)^{2}}{2 \sigma_{i}^{2}}\right] \\
= & \frac{1}{m_{0} \sigma_{i} \sqrt{2 \pi}} \exp \left[-\frac{\bar{m}_{i}}{m_{0}}\left(1-\frac{\sigma_{i}^{2}}{2 m_{0} \bar{m}_{i}}\right)\right] \\
& \times \int_{\bar{m}=0}^{\infty} d \bar{m} \exp \left[-\frac{\left[\bar{m}-\left(\bar{m}_{i}-\sigma_{i}^{2} / m_{0}\right)\right]^{2}}{2 \sigma_{i}^{2}}\right]
\end{aligned}
$$

where, to obtain the second expression, we have completed the square in the exponent of the integrand. The last integral can be calculated analytically, yielding

$$
\begin{aligned}
\ell_{i}= & \frac{1}{2 m_{0}} \exp \left[-\frac{\bar{m}_{i}}{m_{0}}\left(1-\frac{\sigma_{i}^{2}}{2 m_{0} \bar{m}_{i}}\right)\right] \\
& \times\left\{1+\operatorname{erf}\left[\frac{\bar{m}_{i}}{\sigma_{i} \sqrt{2}}\left(1-\frac{\sigma_{i}^{2}}{m_{0} \bar{m}_{i}}\right)\right]\right\} .
\end{aligned}
$$

If we want (as is the case for our data set) to implement data cuts that restrict the values of $\bar{m}_{i}$ to be larger than some limiting value $m_{l}$, the likelihood of a single observation of a modulation index $\bar{m}_{i}$ will be the expression above multiplied by a Heaviside step function, and renormalized so that the likelihood $\ell_{i \text {, cuts }}$ to obtain any value of $\bar{m}_{i}$ above $m_{l}$ is 1 :

$$
\ell_{i, \mathrm{cuts}}\left[m_{l}\right]=\frac{H\left(\bar{m}_{i}-m_{l}\right) \ell_{i}}{\int_{\bar{m}_{i}=m_{l}}^{\infty} d \bar{m}_{i} \ell_{i}} .
$$

This renormalization enforces that there is no probability density for observed events "leaking" in the parameter space of rejected $\bar{m}_{i}$ values. In this way, it "informs" the likelihood that the reason why no objects of $\bar{m}_{i}<m_{l}$ are observed is not because such objects are not found in nature, but rather because we have excluded them "by hand." 


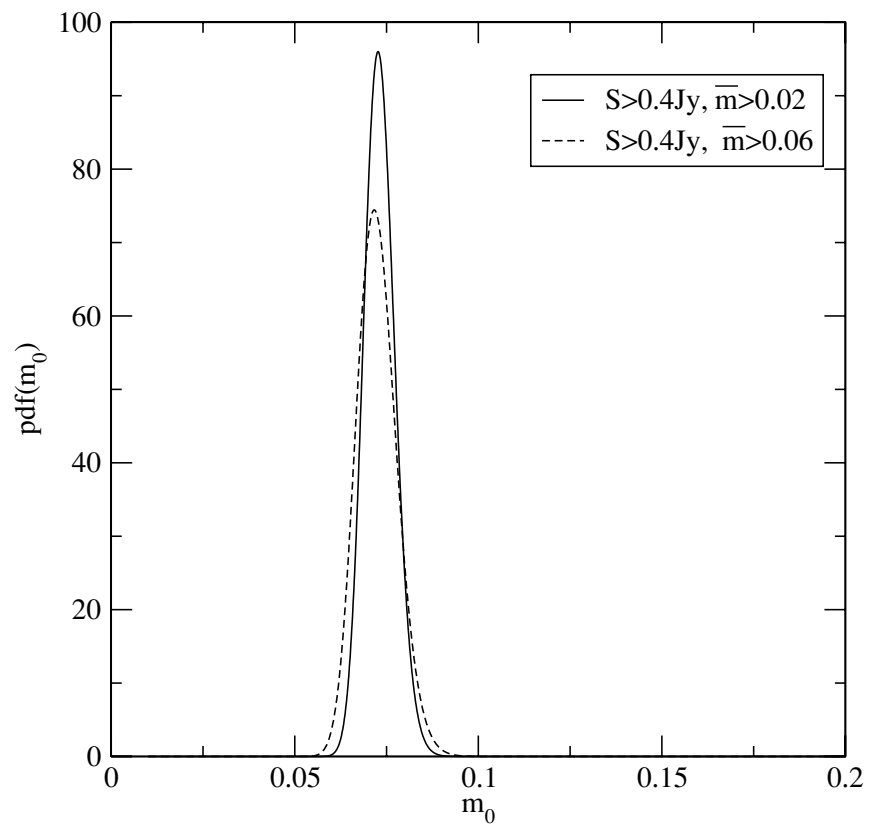

Figure 14. Probability density of $m_{0}$ for the subset of bright CGRaBS blazars not found in 1LAC, for two values of the cutoff for data acceptance: $m_{l}=0.02$ (solid line, maximum-likelihood value and $1 \sigma$ error $m_{0}=0.073 \pm 0.004$ ) and $m_{l}=0.06$ (dashed line, maximum-likelihood value and $1 \sigma$ error $m_{0}=$ $0.072_{-0.005}^{+0.006}$. The two distributions are consistent with a single value.

The integral in the denominator is analytically calculable,

$$
\begin{aligned}
\int_{\bar{m}_{i}=m_{l}}^{\infty} d \bar{m}_{i} \ell_{i}= & \frac{1}{2}\left\{\exp \left(\frac{\sigma_{i}^{2}}{2 m_{0}^{2}}-\frac{m_{l}}{m_{0}}\right)\right. \\
& \times\left[1+\operatorname{erf}\left(\frac{m_{l}}{\sigma_{i} \sqrt{2}}-\frac{\sigma_{i}}{m_{0} \sqrt{2}}\right)\right] \\
& \left.+1-\operatorname{erf}\left(\frac{m_{l}}{\sigma_{i} \sqrt{2}}\right)\right\}
\end{aligned}
$$

The likelihood of $N$ observations of this type is

$$
\mathcal{L}\left(m_{0}\right)=\prod_{i=1}^{N} \ell_{i, \mathrm{cuts}}\left[m_{l}\right]
$$

If we wish to study two parts of the $S_{0}$ parameter space with different cuts (as in, for example, Figure 11, where we have a cut of $m_{l}=0.02$ for $S_{0}>0.4 \mathrm{Jy}$, and a different cut of $m_{u}=0.06$ for $0.06 \mathrm{Jy} \leqslant S_{0} \leqslant 0.4 \mathrm{Jy}$ ), we can implement this in a straightforward way, by considering each segment of the $S_{0}$ parameter space as a distinct "experiment," with its own data cut. If the first "experiment" involves $N_{l}$ objects surviving the $m_{l}$ cut, and the second "experiment" involves $N_{u}$ objects surviving the $m_{u}$ cut, then the overall likelihood will simply be

$$
\mathcal{L}\left(m_{0}\right)=\prod_{i=1}^{N_{l}} \ell_{i, \mathrm{cuts}}\left[m_{l}\right] \prod_{i=1}^{N_{u}} \ell_{i, \mathrm{cuts}}\left[m_{u}\right]
$$

Maximizing Equation (33) we obtain the maximum-likelihood value of $m_{0}, m_{0, \operatorname{maxL}}$. Statistical uncertainties on this value can also be obtained in a straight-forward way, as Equation (33), assuming a flat prior on $m_{0}$, gives the probability density of the mean intrinsic modulation index $m_{0}$ of the subset under study.

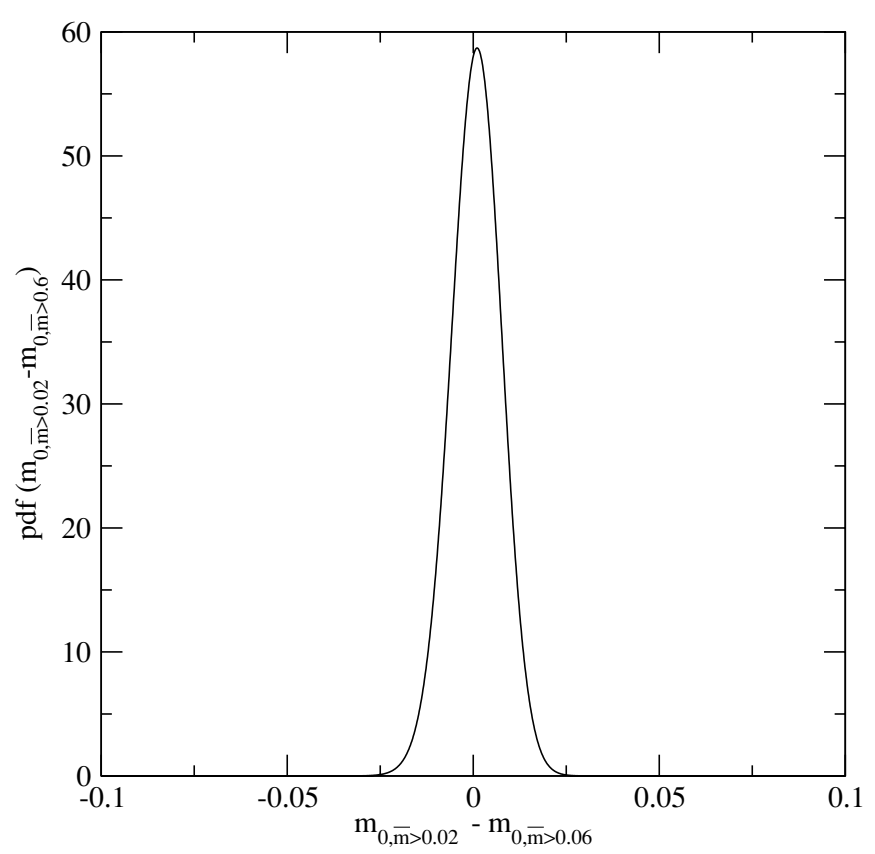

Figure 15. Probability density of the difference between the mean modulation index $m_{0}$ for the two sets considered in Figure 14 . The difference $(0.001 \pm 0.007)$ is consistent with zero within $1 \sigma$.

\subsubsection{Variability Analysis-Population Studies-Results}

Here, we apply the formalism introduced in Section 6.3.3 to examine whether the intrinsic modulation index $\bar{m}$ correlates with the physical properties of the sources in our sample. We will be testing whether the distributions of $\bar{m}$-values in subsets of our monitoring sample split according to some source property are consistent with each other. To verify that our analysis does not yield spurious results, we first discuss three test cases where the likelihood analysis should not find a difference in the variability properties of the different subsets considered.

The first case tests whether the data cuts discussed in Section 6.3.2 are implemented correctly in Section 6.3.3. To this end, we calculate $\mathcal{L}\left(m_{0}\right)$ for the set of non gamma-ray-loud CGRaBS blazars (blazars not found in 1LAC) in our monitoring sample with $S>0.4 \mathrm{Jy}$, in two different ways: first, by applying an $\bar{m}$ cut at $m_{l}=0.02$; and second, by applying an $\bar{m}$ cut at $m_{l}=0.06$ (a much more aggressive cut than necessary for the particular bright blazar population). The increased value of $m_{l}$ in the second case should not affect the result other than by reducing the number of data points and thus resulting in a less constraining likelihood for $m_{0}$. This is indeed the case, as we see in Figure 14, where we plot the probability density of $m_{0}$ for the two subsets. That the two distributions are consistent with each other is explicitly demonstrated in Figure 15, where we plot the probability density of the difference between the means $m_{0}$ of the two subsets (which is formally equal to the cross-correlation of their individual distributions). The difference is consistent with zero within $1 \sigma$.

The second case tests whether a split according to a source property without physical meaning and with the same value for the cutoff modulation index $m_{l}$ will yield probability densities for the $m_{0}$ that are consistent with each other. For this reason, we split the population of bright $(S>0.4 \mathrm{Jy})$ CGRaBS blazars in our monitoring sample in two subsets in the following way: we divide the R.A. of each source by 1 minute. If the remainder of this operation is $<30 \mathrm{~s}$, we include this source 


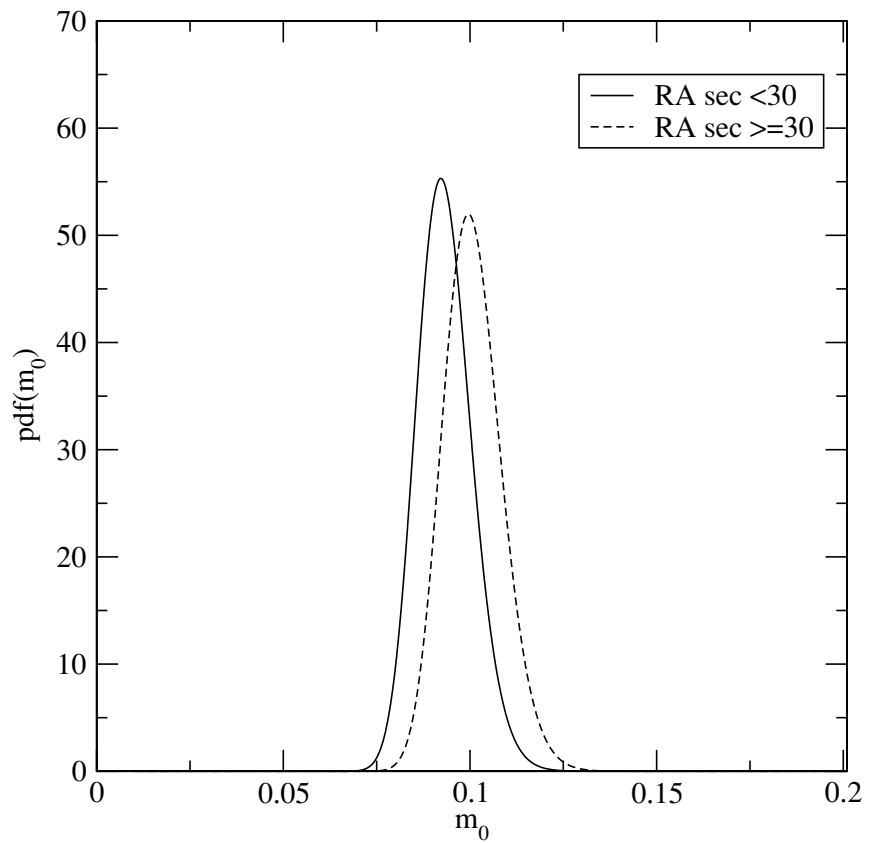

Figure 16. Probability density of $m_{0}$ for the subset of bright CGRaBS blazars: those with seconds of R.A. $<30$ s (solid line, maximum-likelihood value and $1 \sigma$ error $m_{0}=0.088 \pm 0.006$ ) or $\geqslant 30 \mathrm{~s}$ (dashed line, maximum-likelihood value and $1 \sigma$ error $m_{0}=0.096_{-0.006}^{+0.007}$ ). The two distributions are consistent with a single value.

in the first subsample (depicted by a solid line in Figure 16). If the remainder is $\geqslant 30 \mathrm{~s}$ we include the source in the second subsample (depicted by a dashed line in Figure 16). As expected, the probability distributions of $m_{0}$ for the two subsamples, shown in Figure 16, are consistent with each other. This is also explicitly demonstrated in Figure 17, which shows the probability density of the difference between the $m_{0}$ in the two subsamples. The difference is consistent with zero within $1 \sigma$.

In the final test case, we examine whether a split in galactic latitude yields consistent probability densities for the two subsamples. Again, we expect consistent distributions because this division does not reflect a physical property of the sources. For this test, we restrict the sample to bright $(S>0.4 \mathrm{Jy})$ FSRQs and use the cutoff modulation index $m_{l}=0.02$. We split between low- and high-galactic latitude at $|b|=39^{\circ}$. This produces similarly sized subsamples (181 and 168 for low and high latitudes, respectively). Figure 18 shows the probability distributions for $m_{0}$ for these two subsamples, which, as anticipated, are consistent with each other. Figure 19 shows the probability density for the difference between $m_{0}$ for the two subsamples, which is consistent with zero to within $1 \sigma$.

We next examine subsets defined according to physical properties of the sources. The first criterion we apply is whether the source has been detected by Fermi LAT at a significance level high enough to warrant inclusion in the 1LAC catalog. For sources with $S_{0}<0.4 \mathrm{Jy}$ we apply a cut $\bar{m}>m_{u}=0.06$ and for sources with $S_{0} \geqslant 0.4 \mathrm{Jy}$ a cut $\bar{m}>m_{l}=0.02$. The results are shown in Figures 20 and 21. The set of sources that are included in 1LAC is depicted by a solid line, while the set of sources that are not in 1LAC is depicted by a dashed line. The two are not consistent with each other at a confidence level of $6 \sigma$ (Figure 21), with a maximum-likelihood difference of 5.7 percentage points, with gamma-ray-loud blazars exhibiting, on average, a higher variability amplitude by almost a factor of two versus non-gamma-ray-loud blazars.

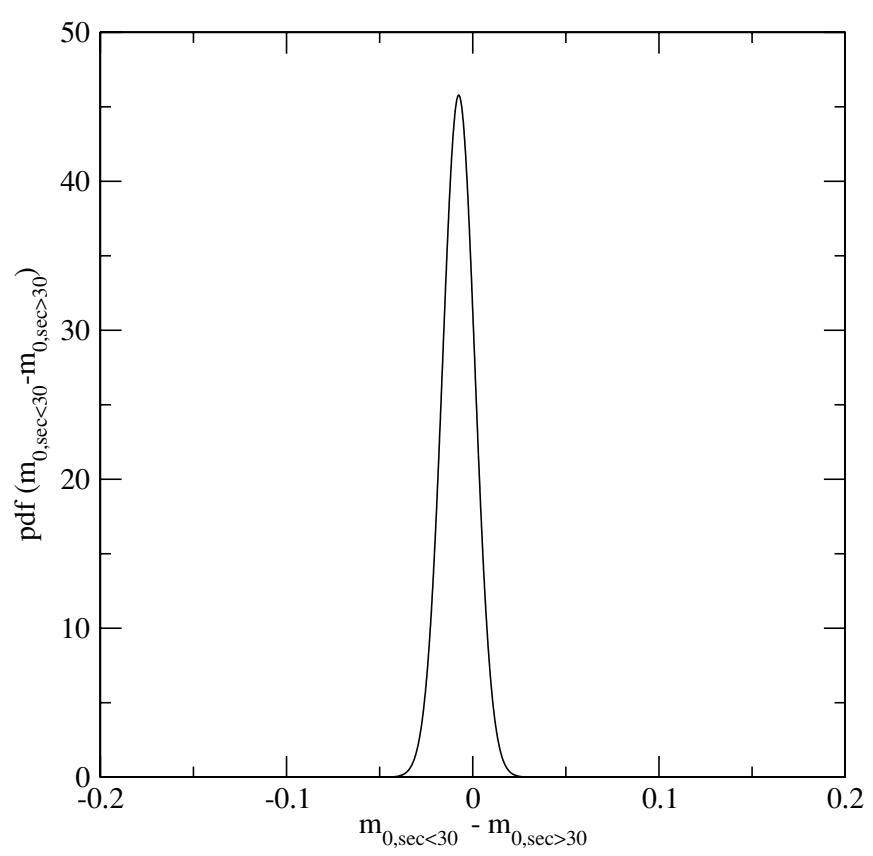

Figure 17. Probability density of the difference between the mean modulation index $m_{0}$ for the two sets considered in Figure 16. The difference $(-0.008 \pm$ 0.009 ) is consistent with zero within $1 \sigma$.

We also examine the variability amplitude properties as a function of optical spectral classification. We analyze the subsets of CGRaBS BL Lacs and FSRQs. The probability densities for the mean $m_{0}$ of the two subsets are shown in Figure 22. The results for BL Lacs (FSRQs) are plotted as a solid (dashed) line. The two curves are not consistent with each other-the BL Lacs appear to have, on average, higher variability amplitude than the FSRQs. We verify this finding by plotting, in Figure 23, the probability density of the difference between the $m_{0}$ of BL Lacs and FSRQs. The most likely difference is 3.2 percentage points, and it is more than $3 \sigma$ away from zero. Note that the difference between BL Lacs and FSRQs is less significant than that between gamma-ray-loud and non gamma-ray-loud blazars. This is both because the most likely difference in $m_{0}$ values between the BL Lac and FSRQ subsets is smaller and because the BL Lac sample is smaller than the gamma-ray-loud blazar sample: only 94 BL Lacs satisfy the data cuts we impose, versus 191 gamma-ray-loud blazars. As a result, the constraints on the intrinsic distribution of modulation indices (i.e., on $m_{0}$ ) are stronger in the latter case.

Finally, we examine the dependence of variability amplitude on redshift. In Figure 24 we plot the mean $\bar{m}$ (as calculated by a simple average rather than the likelihood analysis) in redshift bins of $\Delta z=0.5$ for bright ( $S \geqslant 0.4$ Jy) FSRQs with known redshifts in our monitoring sample. We exclude BL Lacs from this analysis so as not to bias the result, as BL Lacs with known redshifts are located at low $z$, and we have also already shown that they have a higher mean $\bar{m}$ compared to FSRQs. Although the errors are large, there is a hint of a trend toward decreasing variability amplitude with increasing redshift. We further test the significance of this result by splitting sources in our monitored sample in high- and low-redshift subsets with the dividing redshift at $z=1$ (dashed line in Figure 24). In the two subsets we also include faint $(S<0.4$ Jy) sources, with the usual cut at $m_{u}=0.06$. The probability density for the mean $m_{0}$ of each subset is shown in Figure 25, where the solid curve corresponds to low-redshift blazars and the dashed 


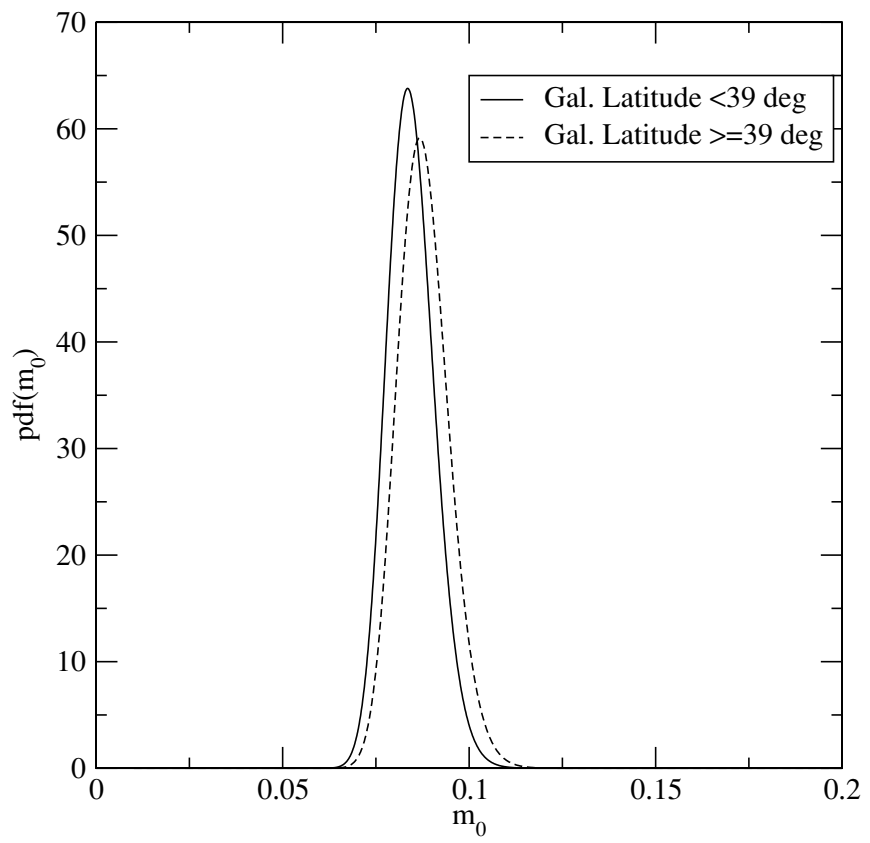

Figure 18. Probability density of $m_{0}$ for the subset of bright $(S>0.4 \mathrm{Jy})$ FSRQs with $\bar{m}>0.02$ : those at low-galactic latitude $\left(|b|<39^{\circ}\right.$, solid line, maximumlikelihood value, and $1 \sigma$ error $\left.m_{0}=0.084_{-0.006}^{+0.007}\right)$ or high-galactic latitude $(|b| \geqslant$ $39^{\circ}$, dashed line, maximum-likelihood value, and $1 \sigma$ error $m_{0}=0.087_{-0.006}^{+0.007}$ ). The two distributions are consistent with a single value.

curve to high-redshift FSRQs. We find that low-redshift FSRQs have higher, on average, intrinsic modulation indices. The result is shown to be statistically significant in Figure 26, where we plot the probability density of the difference between $m_{0}$ in each subset. The most likely difference is found to be about 2.4 percentage points, and more than $3 \sigma$ away from zero.

\section{DISCUSSION AND CONCLUSIONS}

We have discussed in detail the OVRO 40 m telescope $15 \mathrm{GHz}$ monitoring program. We have presented results from the first two years of observations, including reduced data and light curves for all sources in our monitoring sample.

We have derived the variability amplitude properties of all blazars in our sample through a likelihood analysis that deconvolves the intrinsic variability from scatter induced due to errors in individual flux density measurements and accounts for uncertainties due to finite (and different) sampling in each source to calculate an intrinsic modulation index as well as uncertainties on its value. We have used these intrinsic modulation indices to study whether and how the variability amplitude is correlated with physical properties of our sources.

We have found that the distribution of intrinsic modulation indices is different between sources that have/have not been detected by Fermi in GeV gamma rays, between BL Lacs/ FSRQs, and between FSRQs at high and low redshifts.

Our most significant result is that gamma-ray-loud sources have a higher, on average, variability amplitude, as quantified by the intrinsic modulation indices, than non-gamma-ray-loud sources. The most likely difference in mean modulation index is about 5.7 percentage points, so that gamma-ray-loud sources have, on average, a variability amplitude almost a factor of two higher than sources not found in 1LAC. The result is very significant statistically, with the maximum-likelihood difference being $6 \sigma$ away from 0 .

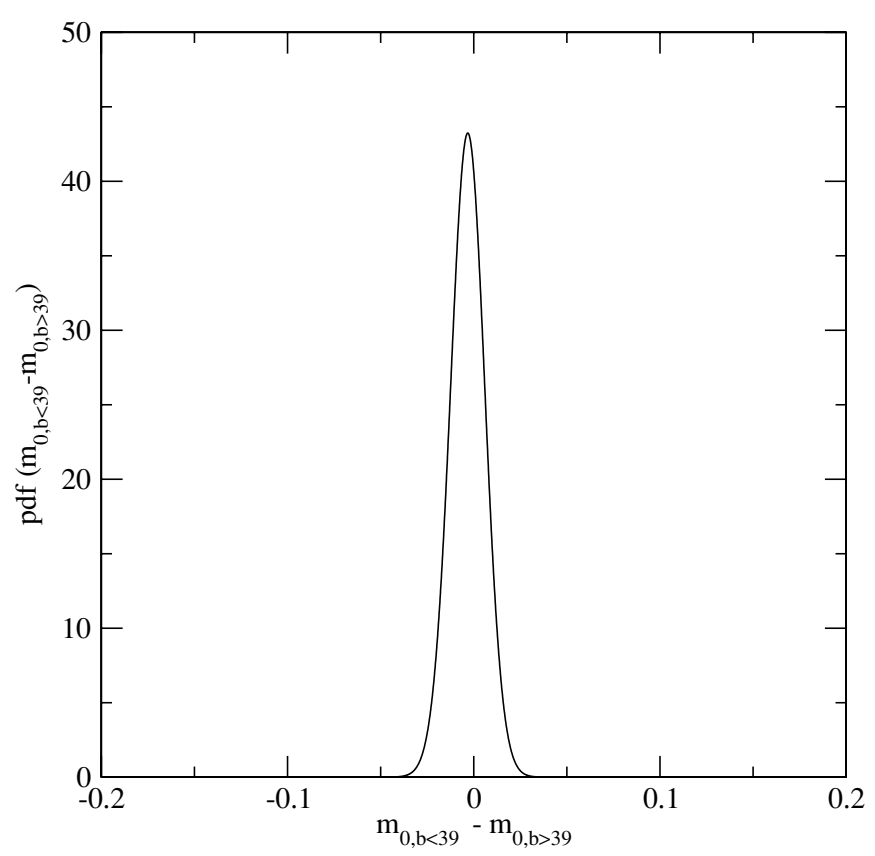

Figure 19. Probability density of the difference between the mean modulation index $m_{0}$ for the two sets considered in Figure 18. The difference $(-0.003 \pm$ 0.009 ) is consistent with zero within $1 \sigma$.

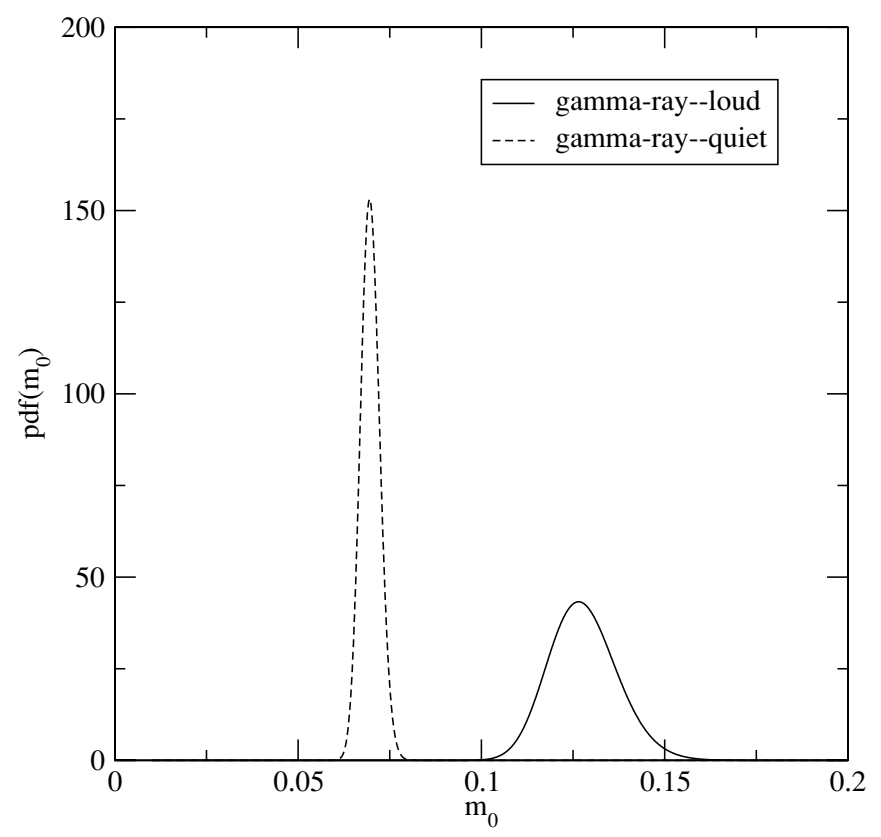

Figure 20. Probability density of $m_{0}$ for CGRaBS blazars in our monitoring sample that are (solid line, maximum-likelihood value and $1 \sigma$ error $m_{0}=$ $0.127_{-0.009}^{+0.010}$ ) and are not (dashed line, maximum-likelihood value and $1 \sigma$ error $m_{0}=0.070 \pm 0.003$ ) included in 1LAC. The two distributions are not consistent with a single value.

It is not clear whether a selection effect or an intrinsic difference is responsible for this deviation between the two subsets. It is, for example, conceivable, that all CGRaBS blazars are potentially gamma-ray-loud at some part of their activity cycle and, given enough observation time, all of them would enter their "flaring" state (that would presumably be characterized by enhanced broadband luminosity, including increased flux density at $15 \mathrm{GHz}$ ) and would be detected in $\mathrm{GeV}$ gamma rays. If this is the case, then the blazars that have been detected by Fermi so far would be the ones that happened 


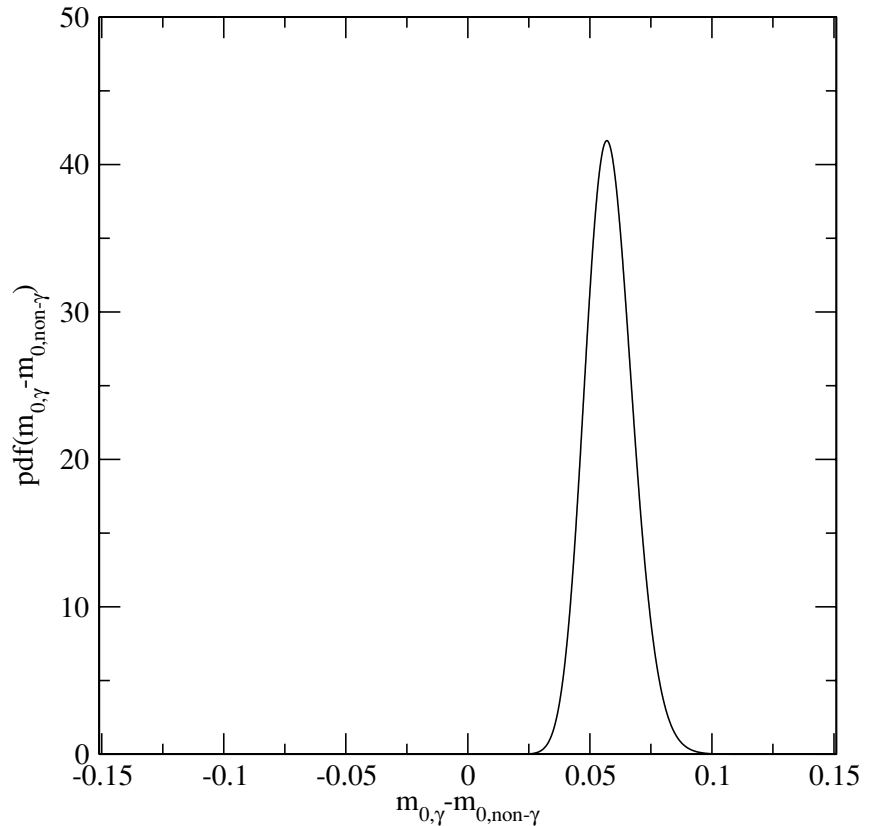

Figure 21. Probability density of the difference between the mean modulation index $m_{0}$ for the two sets considered in Figure 20. The peak of the distribution $\left(0.057_{-0.009}^{+0.010}\right)$ is $6 \sigma$ away from zero.

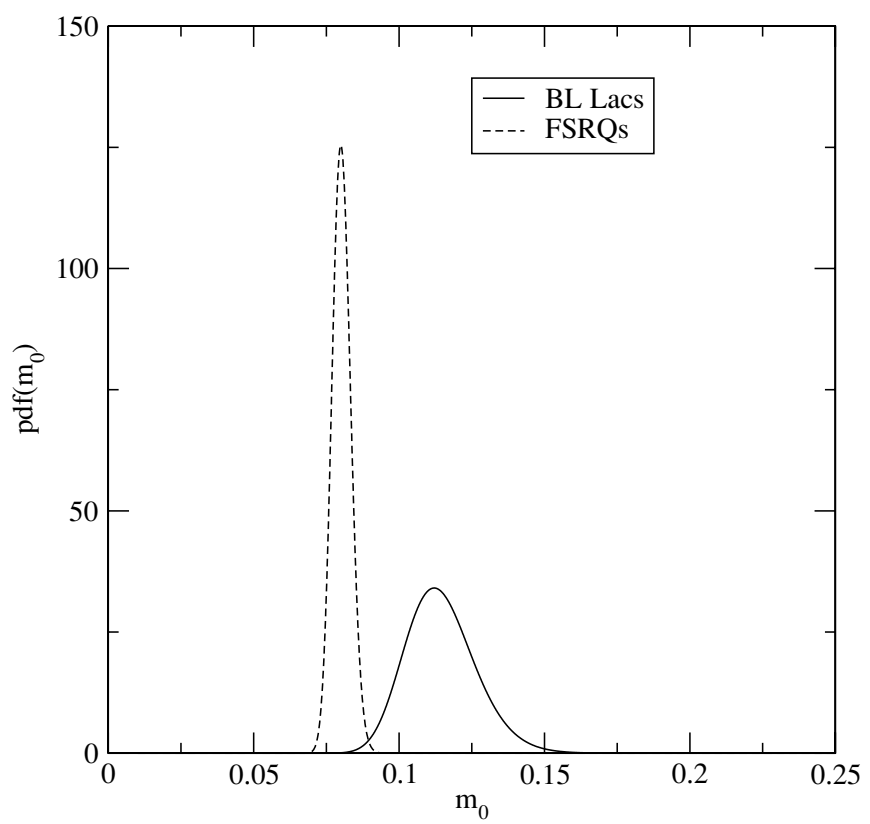

Figure 22. Probability density of $m_{0}$ for BL Lac (solid line, maximum-likelihood value and $1 \sigma$ error $m_{0}=0.112_{-0.011}^{+0.013}$ ) and FSRQ (dashed line, maximumlikelihood value and $1 \sigma$ error $m_{0}=0.080 \pm 0.003$ ) CGRaBS blazars in our monitoring sample. The two distributions are not consistent with a single value.

to have been in their "flaring" state during the first year of Fermi operations, and it would be expected that they are seen to have a higher, on average, variability amplitude in $15 \mathrm{GHz}$ as well. In this scenario, given more time, more blazars in our sample will enter at some point their "flaring" state; they will be detected in gamma rays, and the amplitude of their $15 \mathrm{GHz}$ emission will also increase. If we were to repeat the same experiment after another two years of observations, the source numbers in the two subsamples would change, but not the average population properties: more sources would be detected in gamma rays, but these sources would now also exhibit a higher $\bar{m}$. The average $m_{0}$

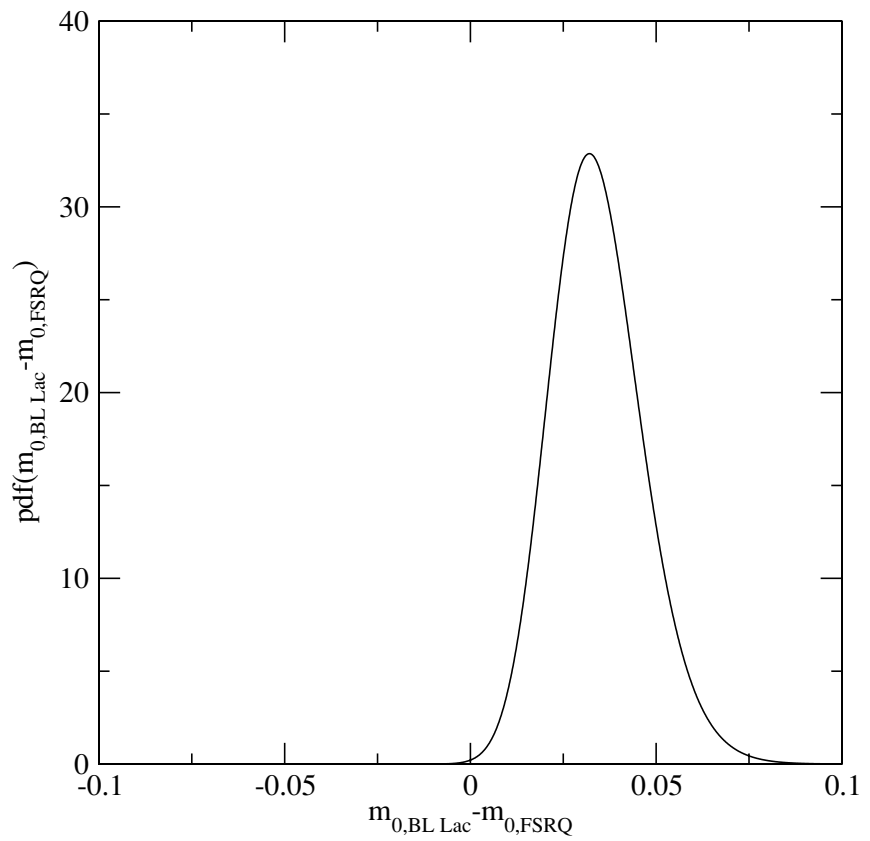

Figure 23. Probability density of the difference between the mean modulation index $m_{0}$ for the two sets considered in Figure 22. The peak of the distribution $\left(0.032_{-0.011}^{+0.013}\right)$ is more than $3 \sigma$ away from zero.

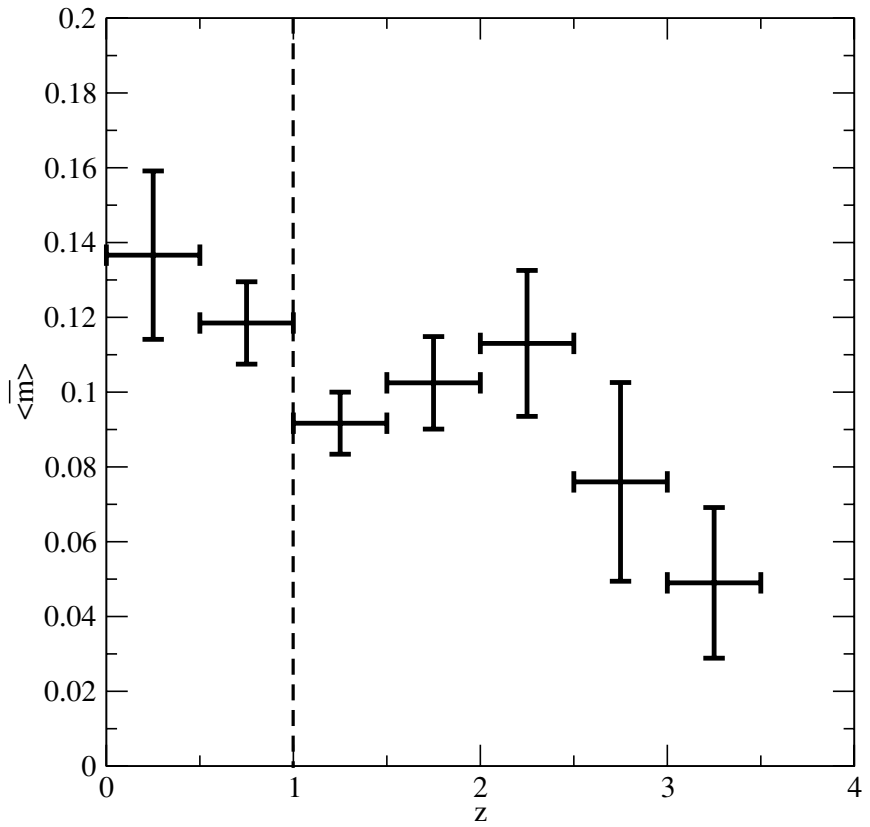

Figure 24. Mean $\bar{m}$ in redshift bins of 0.5 for bright $(S>0.5$ Jy) FSRQs in our monitoring sample.

of each population would not change appreciably, but sources would move from one category (non-gamma-ray-loud) to the other (gamma-ray-loud). If on the other hand we have seen all blazars in our monitoring sample in all activity states, then the variability amplitudes of each are not expected to change appreciably if we observe them for longer, and the number statistics in the two categories will likely remain fixed (for a fixed gamma-ray flux detection threshold). In this scenario, the variability amplitude is the result of some intrinsic, persistent physical property of blazars, which is also related to the gammaray activity of the source.

BL Lacs are found to have higher, on average, intrinsic modulation indices than FSRQs, by about 3.2 percentage points. 


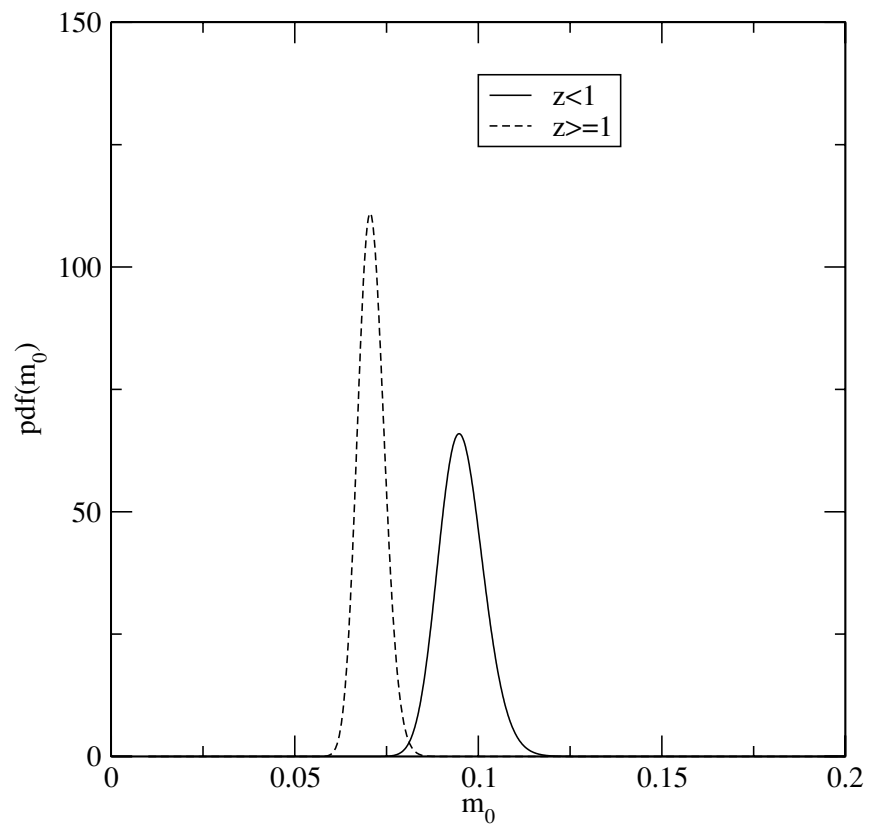

Figure 25. Probability density of $m_{0}$ for FSRQs in our monitoring sample with $z<1.0$ (solid line, maximum-likelihood value and $1 \sigma$ error $m_{0}=$ $0.095 \pm 0.006$ ) and $z \geqslant 1.0$ (dashed line, maximum-likelihood value and $1 \sigma$ error $m_{0}=0.071 \pm 0.004$ ). The two distributions are not consistent with a single value.

Due to the smaller-number statistics and on-average smaller difference variability, the difference is less significant, but still more than $3 \sigma$ away from 0 . This trend of higher variability amplitude among BL Lacs than among FSRQs agrees with results from previous radio variability studies (e.g., Aller et al. 1999). Our larger sample and statistical method allows us to demonstrate the statistical significance of this result.

In addition, among our FSRQ subsample, low-redshift sources are found to have higher, on average, intrinsic modulation indices, with the most likely difference on the mean being about 2.4 percentage points, also more than $3 \sigma$ away from 0 . This difference is not easy to interpret as an indication of source evolution, as there are competing effects that could affect the result in either way. On the one hand, sources at higher redshift have been observed for a shorter rest-frame time interval due to time-dilation effects, so it is conceivable that high-redshift sources have not been followed through their complete activity cycles, and their intrinsic modulation indices will increase as they are observed for longer. On the other hand, sources at higher redshift are being observed at a higher rest-frame frequency. Because the radio variability amplitude increases with increasing frequency, this effect should yield higher modulation indices for higher-redshift sources. As our monitoring program is continued, the importance of the first effect will decrease (or, conversely, with long enough light curves, we could select to look at shorter light curve segments for our low-redshift sources, corresponding to the same rest-frame time interval as for our highest-redshift sources). The second effect is not affected by length of observation time, however it operates in the opposite direction to the observed effect. Should the trend persists as it is seen here (low-redshift sources have higher modulation indices), this might be an indication of source evolution with cosmic time toward higher variability amplitudes.

We have found larger variability for the LAT-detected blazars, for the BL Lac-type blazars, and for the sources at lower redshift. In addition, we have noted the relatively modest overlap

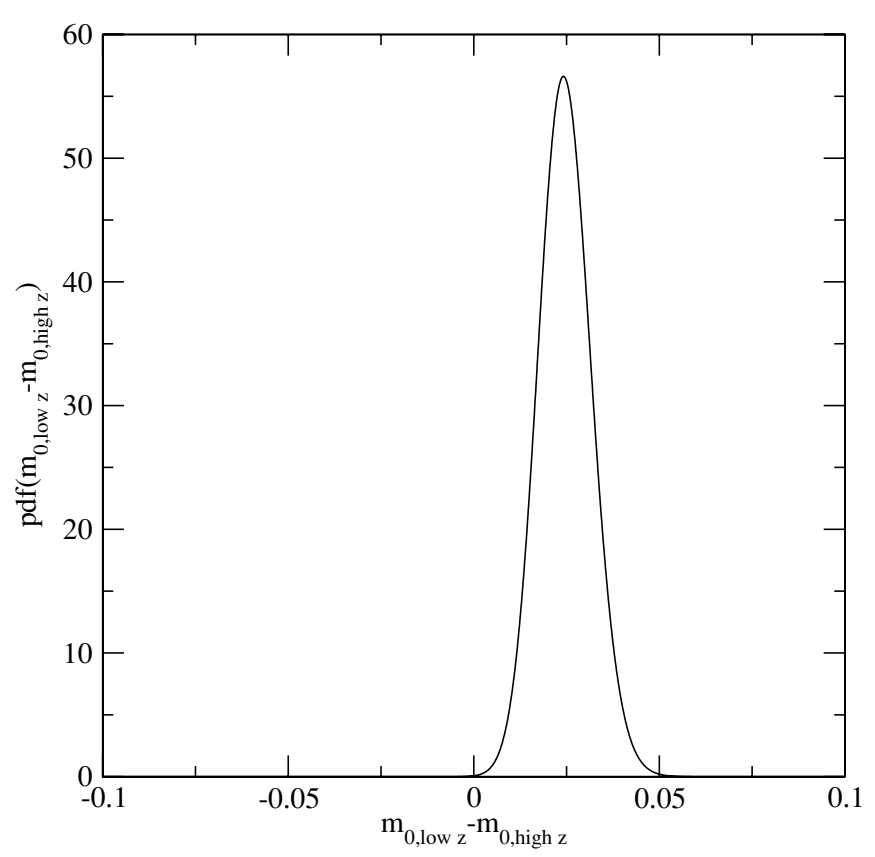

Figure 26. Probability density of the difference between the mean modulation index $m_{0}$ for the two sets considered in Figure 25. The peak of the distribution $(0.024 \pm 0.007)$ is more than $3 \sigma$ away from zero.

between the (EGRET-like) CGRaBS sources and the early LAT detections. These facts may be related: it has already been shown in Abdo et al. (2010b) that the decrease in LAT effective area below $0.3 \mathrm{GeV}$ has strongly biased the LAT detections to the relatively hard-spectrum high-peak blazars, especially the $\mathrm{BL}$ Lacs, compared to the EGRET sample. Indeed 1LAC contained $\sim 50 \%$ BL Lacs, while for EGRET FSRQs outnumbered BL Lacs by $>3 \times$. These BL Lacs are radio fainter and tend to be lower-power sources at relatively low redshift. Thus, we expect from the higher variability amplitude found for BL Lacs and lower- $z$ sources in this paper that the LAT-detected blazar sample should have higher average variability. We note however that the difference in variability amplitude between gamma-rayloud and gamma-ray-quiet blazars in our sample is much larger than the difference between BL Lacs and FSRQs, so this effect cannot be attributed in its entirety to different BL Lac/FSRQ number ratios in the CGRaBS and LAT-detected blazar samples. As LAT exposure increases and as refinement of the event cuts allows more effective area at lower energy, we might expect an increase in high-power, high-redshift FSRQ detections, with steeper gamma-ray spectral indices. These sources would have variability of lower amplitude and/or longer observed timescale. Indeed, continued LAT exposure is detecting more CGRaBS sources and we expect that our OVRO-monitored sample will allow an excellent comparison of radio variability statistics on rest-frame timescales comparable to those now probed for the nearby BL Lacs.

In conclusion, we have, for the first time, been able to explicitly demonstrate that the radio variability amplitude of blazars exhibits positive, statistically significant correlations with physically meaningful properties of the sources. Our findings are important steps toward understanding the physical differences between blazars with otherwise similar properties which, however, differ in their gamma-ray activity.

The variability amplitude in radio frequencies has never before been considered as a differentiating property when constructing blazar samples; this was largely due to practical 
purposes, as never before has such a large, preselected, statistically complete sample been monitored for as long a time and with as high a cadence. The compilation of the CGRaBS sample (Healey et al. 2008), for example, was based on radio flux density, radio spectral index, and X-ray flux; variability information was not included, not because it was not considered important, but rather because such information was, at the time, unavailable. As a result, the CGRaBS catalog had only moderate success in predicting sources that would emerge as gamma-ray sources in the LAT era. However, by providing a preselected sample defined by robust statistical criteria, the CGRaBS sample has allowed us to make unprecedented progress in studying the population properties of blazars, as in this work.

As the additional, non-CGRaBS blazars that have been discovered by Fermi have now been added to our monitored source sample, our program will allow us to confirm and expand these results in upcoming years. In addition, by establishing, through the results of this work as well as those presented in V. Pavlidou et al. (2011, in preparation) and A. Abdo et al. (2011, in preparation), that there is a close connection between gamma-ray and $15 \mathrm{GHz}$ blazar emission, we are justified to expect that additional progress in blazar jet physics is to be expected through cross-correlations in the time domain between $15 \mathrm{GHz}$ and Fermi-LAT gamma-ray light curves. Such crosscorrelations will be discussed in an upcoming publication (W. Max-Moerbeck, et al. 2011, in preparation).

We are grateful to Russ Keeney for his tireless efforts in support of observations at the Owens Valley Radio observatory. The OVRO $40 \mathrm{~m}$ monitoring program is supported in part by NASA grants NNX08AW31G and NNG06GG1G and NSF grant AST-0808050. Support from the Max-Planck Institut für Radioastronomie for upgrading the OVRO $40 \mathrm{~m}$ telescope receiver is also acknowledged. O.G.K. acknowledges the support of a Keck Institute for Space Studies Fellowship. W.M. acknowledges support from the US Department of State and the Comisión Nacional de Investigación Científica y Tecnológica (CONICYT) in Chile for a Fulbright-CONICYT scholarship. V.P. acknowledges support for this work provided by NASA through Einstein Postdoctoral Fellowship grant number PF890060 awarded by the Chandra X-ray Center, which is operated by the Smithsonian Astrophysical Observatory for NASA under contract NAS8-03060, and thanks the Department of Physics at the University of Crete for their hospitality during the completion of part of this work.

Facilities: OVRO:40m

\section{REFERENCES}

Abdo, A. A., et al. 2009, ApJ, 699, 976

Abdo, A. A., et al. 2010a, ApJS, 188, 405

Abdo, A. A., et al. 2010b, ApJ, 715, 429
Acciari, V. A., et al. 2009, Science, 325, 444

Acciari, V. A., et al. 2010, ApJ, 709, L163

Aharonian, F., et al. 2007, ApJ, 664, L71

Aharonian, F., et al. 2009, A\&A, 502, 749

Aller, M. F., Aller, H. D., \& Hughes, P. A. 1992, ApJ, 399, 16

Aller, M. F., Aller, H. D., \& Hughes, P. A. 2003, ApJ, 586, 33

Aller, M. F., Aller, H. D., Hughes, P. A., \& Latimer, G. E. 1999, ApJ, 512, 601

Angelakis, E., Fuhrmann, L., Nestoras, I., Zensus, J. A., Marchili, N., Pavlidou, V., \& Krichbaum, T. P. 2010, in Proc. Workshop Fermi meets Jansky: AGN in Radio and Gamma-Rays, ed. T. Savolainen, E. Ros, R. W. Porcas, \& J. A. Zensus (Bonn: MPIfR), 81 (http://www.mpifr-bonn.mpg.de/div/vlbi/agn2010)

Angelakis, E., Kraus, A., Readhead, A. C. S., Zensus, J. A., Bustos, R., Krichbaum, T. P., Witzel, A., \& Pearson, T. J. 2009, A\&A, 501, 801 Atwood, et al. 2009, ApJ, 697, 1071

Baars, J. W. M., Genzel, R., Pauliny-Toth, I. I. K., \& Witzel, A. 1977, A\&A, 61,99

Blandford, R. D., \& Levinson, A. 1995, ApJ, 441, 79

Blandford, R. D., \& Payne, D. G. 1982, MNRAS, 199, 883

Blandford, R. D., \& Rees, M. J. 1974, MNRAS, 169, 395

Blandford, R. D., \& Znajek, R. L. 1977, MNRAS, 179, 433

Dennett-Thorpe, J., \& de Bruyn, A. G. 2000, ApJ, 529, L65

Dermer, C. D., Schlickeiser, R., \& Mastichiadis, A. 1992, A\&A, 256, L27

Edelson, R., Turner, T. J., Pounds, K., Vaughan, S., Markowitz, A., Marshall, H., Dobbie, P., \& Warwick, R. 2002, ApJ, 568, 610

Fermi-LAT Collaboration, et al. 2010, Nature, 463, 919

Fuhrmann, L., Zensus, J. A., Krichbaum, T. P., Angelakis, E., \& Readhead, A. C. S. 2007, in AIP Conf. Ser. 921, The First GLAST Symposium, ed. S. Ritz, P. Michelson, \& C. A. Meegan (Melville, NY: AIP), 249

Górski, K. M., Hivon, E., Banday, A. J., Wandelt, B. D., Hansen, F. K., Reinecke, M., \& Bartelmann, M. 2005, ApJ, 622, 759

Healey, S. E., et al. 2008, ApJS, 175, 97

Jauncey, D. L., Kedziora-Chudczer, L. L., Lovell, J. E. J., Nicolson, G. D., Perley, R. A., Reynolds, J. E., Tzioumis, A. K., \& Wieringa, M. H. 2000, in Astrophysical Phenomena Revealed by Space VLBI, ed. H. Hirabayashi, P. G. Edwards, \& D. W. Murphy (Sagamihara: ISAS), 147

Jorstad, S. G., Marscher, A. P., Mattox, J. R., Aller, M. F., Aller, H. D., Wehrle, A. E., \& Bloom, S. D. 2001, ApJ, 556, 738

Kedziora-Chudczer, L., Jauncey, D. L., Wieringa, M. H., Walker, M. A., Nicolson, G. D., Reynolds, J. E., \& Tzioumis, A. K. 1997, ApJ, 490, L9

Kraus, A., et al. 2003, A\&A, 401, 161

Lilliefors, H. W. 1967, J. Am. Stat. Assoc., 62, 399

Lovell, J. E. J., et al. 2008, ApJ, 689, 108

Macquart, J., \& de Bruyn, A. G. 2007, MNRAS, 380, L20

Mannheim, K. 1993, A\&A, 269, 67

Marscher, A. P., et al. 2008, Nature, 452, 966

McKinney, J. C., \& Blandford, R. D. 2009, MNRAS, 394, L126

Penna, R. F., McKinney, J. C., Narayan, R., Tchekhovskoy, A., Shafee, R., \& McClintock, J. E. 2010, MNRAS, 408, 752

Readhead, A. C. S., Lawrence, C. R., Myers, S. T., Sargent, W. L. W., Hardebeck, H. E., \& Moffet, A. T. 1989, ApJ, 346, 566

Rickett, B., Kedziora-Chudczer, L., \& Jauncey, D. L. 2002, PASA, 19, 106

Rickett, B. J., Lazio, T. J. W., \& Ghigo, F. D. 2006, ApJS, 165, 439

Rohlfs, K., \& Wilson, T. L. 2000, Tools of Radio Astronomy (New York: Springer), 64

Sikora, M., Begelman, M. C., \& Rees, M. J. 1994, ApJ, 421, 153

Soldi, S., et al. 2008, A\&A, 486, 411

Venters, T. M., \& Pavlidou, V. 2007, ApJ, 666, 128

Waldram, E. M., Pooley, G. G., Davies, M. L., Grainge, K. J. B., \& Scott, P. F 2010, MNRAS, 404, 1005

Wall, J. V., \& Jenkins, C. R. 2003, in Practical Statistics for Astronomers, (Cambridge: Cambridge Univ. Press), 90 